\title{
DOCUMENTATION
}

\section{COMMENT RASSASIER L'APPÉTIT D'UNE VACHE A LAIT}

\author{
PAR
}

Friedrich Ernst FISSMER

(Institut de Recherches pour les questions laitières à Kiel)

(Zeitschrift für Tierernährung und Futtermittelkunde)

Band 5, I94I, pages I à I09

Traduit par André VoISIN

\section{A. - L'ÉVOLUTION DES MÉTHODES D'ALIMENTATION ET LEUR INFLUENCE SUR LE PROBLEME ETUDIÉ DANS CET ARTICLE}

A l'époque où on jugeait un aliment à l'aide de l'unité " valeur foin " on n'était pas très exigeant sur l'alimentation exacte à donner pour obtenir une production déterminée de lait. Les besoins de la vache en éléments nutritifs et en aliments pouvaient être facilement satisfaits avec des rations relativement simples. Quand on employait uniquement les aliments de la ferme dont on n'exigeait pas de fortes richesses en substances nutritives, la question du rassasiement de la vache jouait un rôle très peu important, et n'était guère traitée dans les ouvrages de cette époque. Comme on indiquait par exemple que la ration d'entretien des ruminants était de $1,5-2 \mathrm{~kg} d$ ' " unités foin ", et que la ration totale d'une vache à lait était de $3-3,5 \mathrm{~kg}$ pour Ioo $\mathrm{kg}$ de poids vif (KRAFFT, 28) on peut dire que la question de la satisfaction de l'appétit de la vache était toute réglée.

Mais la question changea quand on commença à juger les aliments d'après leur analyse chimique. LIEBIG (35) commença en I839 à étudier le processus d'alimentation animale. Des chercheurs comme BISchoff et VorT (I), HENNEBERG et STOHMANN (24) développèrent les principes qui aboutirent finalement à la conception de la "valeur amidon " (Stärkewert) de KELLNER.

Cette évolution des théories de l'alimentation amena une augmentation de la production des vaches laitières. Ces productions plus fortes exigèrent des

Nota. - La partie concernant les essais de digestibilité des aliments employés, au moyen de moutons, n'a pas été traduite. 
apports plus élevés de substances nutritives et, par suite, amenèrent une amélioration considérable des méthodes d'alimentation. La question du volume de l'aliment commença à apparaître et on fut amené à considérer la question du rassasiement de la vache.

La solution consiste d'abord, conformément aux indications de KELLNER (26), à donner autant d'aliments de base (Grund futter) (foin, paille, betteraves) qu'il était nécessaire pour satisfaire les besoins d'entretien de la vache. Puis on ajoutait la quantité d'aliments concentrés, achetés à l'extérieur, et qui étaient nécessaires pour satisfaire les besoins correspondant à la production de lait de la vache. Les recherches de MünzBERG et NolTE (43) qui furent poursuivies dans toute 1'Allemagne en 1925-I927 montrèrent que, dans un tiers des fermes, qui n'étaient pas soumises au contrôle laitier, les rations utilisées fournissaient juste les besoins énergétiques d'entretien et que, même la quantité de protéine digestible contenue dans la ration ne suffisait pas à couvrir les besoins d'entretien en protéine. Par contre, dans $3 / 4$ des fermes il était donné des quantités d'unités amidon qui dépassaient de beaucoup les besoins d'entretien ; mais, là encore les efforts de protéine digestible étaient insuffisants. Il en résultait donc que l'alimentation de base ne laissait disponible aucune substance nutritive pour la production du lait. C'était seulement dans 3 à $6 \%$ des fermes que l'alimentation de base contenait suffisamment de substances nutritives pour permettre une production quotidienne de $6 \mathrm{~kg}$ de lait. Si nous manquons de données globales après I927, nous pouvons cependant admettre que presque tout le lait produit l'était en fait grâce aux aliments concentrés. La question du volume de l'aliment et, par suite celle du rassasiement de la vache ne jouaient qu'un rôle secondaire. Cette exigence du rassasiement était très facilement satisfaite du reste, en donnant de la paille aux vaches comme WERNER (54) le recommande.

Mais les conditions alimentaires d'aujourd'hui ont totalement changé. Il est nécessaire d'obtenir de nos vaches une forte production sans leur donner des aliments provenant de l'étranger. Or, les produits de nos fermes, comme le fait remarquer BüNGER (6) se distinguent des aliments concentrés venant de l'étranger en ce qu'ils sont des aliments volumineux, c'est-à-dire que, par unité de volume et de poids, ils contiennent peu de substances digestibles et, par conséquent, sont riches en ballast, richesse qui est surtout fonction de la teneur en fibres.

La vache, pour absorber la même quantité d'éléments nutritifs qui lui étaient fournis auparavant par les aliments concentrés étrangers, devrait réussir à manger une bien plus grande quantité d'aliments. Ce qui nous intérésse est donc de savoir la production limite de lait que nous pouvons obtenir avec les aliments produits dans nos fermes. La question de la détermination du rassasiement de la vache est donc très importante.

C'est pourquoi cette étude a été entreprise.

Nous examinerons d'abord les données que nous trouvons dans la littérature sur la question. 


\section{B. - DONNEES DE LA LITTERATURE SUR LE RASSASIEMENT DE LA VACHE LAITIERE :}

\section{I. - Idées sur la nécessité de créer chez la vache le sentiment d'être rassasiée.}

Dans presque tous les ouvrages et les rapports scientifiques on insiste avant tout sur la nécessité de créer chez la vache le sentiment d'être rassasiée par la nourriture mise à sa disposition.

SETTTEGAST (47) avait déjà indiqué, en I868, dans son livre Tierzucht qu'une alimentation rationnelle doit non seulement fournir à l'animal les substances nutritives nécessaires " mais que 1'appareil digestif doit également recevoit un volume d'aliment suffisant ». SETTEGAST avait déjà reconnu que ce volume doit toujours être le même, que l'aliment serve pour l'entretien seul ou pour la ration de production également.

KELLNER (26, page 407) dit qu'une ration composée rationnellement doit être adaptée à la capacité d'absorption du système digestif. Une ration qui ne rassasie pas l'animal ne remplit pas complètement son but, car l'animal tant qu'il a faim, demeure agité.

Dans sa remise à jour de l'ouvrage de KELLNER, FingERLing (26) indique que dans le calcul d'une ration, il faut considérer avant tout " le volume de l'aliment, 1a teneur en protéine réellement digestible et la valeur totale nutritive ". Le volume de 1'aliment est considéré encore comme plus important que les richesses en substances nutritives.

Honcamp (25) et HANSEN (25) insistent également sur l'importance de donner à la vache une alimentation qui lui permettra de se sentir rassasiée. HonCAMP estime que les ruminants, ne peuvent demeurer en bonne santé et profiter de leur nourriture que " si les aliments grossiers voluminetux forment une partie convenable de l'aliment fourni ". C'est en effet seulement ainsi que peut se réaliser le remplissage indispensable du système digestif, ce qui favorisera les mouvements de l'intestin, lesquels sont indispensables pour faire progresser le bol alimentaire à travers le système digestif.

Avec la nourriture riche en aliments concentrés, complétée par des grosses quantités d'aliments aqueux succulents, il était souvent à crainđre que 1'on ne donne pas toujours à la vache le sentiment d'être rassasiée, car l'apport d'aliments grossiers volumineux était souvent trop faible. WERNER (54) conseille de fournir à la vache autant de paille qu'il en faut pour qu'elle en mange jusqu'à ce qu'elle se sente rassasiée. Le rassasiement, dit WERNER (55), se reconnaît au fait que la vache se couche ; d'après lui, cette attitude est la seule indication permettant de juger du rassasiement. Une définition plus satisfaisante du sentiment de rassasiement n'a pu jusqu'ici être donnée. WERNER, comme FINGERLING, attache une grande importance à l'action rassasiante d'un ali- 
ment, estime que l'on doit scientifiquement juger d'un aliment à deux points de vue, à savoir d'après son action rassasiante et d'après son action nutritive.

MüLLER (42) a étudié la question de l'alimentation des vaches laitières avec des quantités faibles ou même nulles d'aliments grossiers volumineux. Il utilisa deux vaches à lait qui, pendant la période d'essais, reçurent une nourriture sans aliments grossiers volumineux, et qui comprenait uniquement des aliments concentrés et des betteraves. Les vaches recevaient ainsi une quantité suffisante de substances nutritives mais un volume insuffisant d'aliments. MüLLER rapporte que les vaches avaient mangé toute leur ration en quelques minutes et qu'en un quart d'heure les auges étaient léchées : "Les bêtes exprimaient le désir de recevoir plus d'aliments en mugissant chaque fois qu'une personne se trouvait dans l'étable. S'il n'y avait personne, elles se calmaient et se couchaient de manière normale pour se reposer ". Mais les bêtes ne ruminaient plus de la manière habituelle; par contre, on observait (rarement du reste) que les vaches rotaient, sans que cependant le ruminement se produise. Avec le temps, ces deux vaches s'habituèrent à ce volume moins important d'aliments, et à la fin de la période d'essai elles étaient plus tranquilles qu'au début.

$\mathrm{Au}$ cours des essais le bousat des vaches resta relativement ferme.

A la suite de ces recherches, Mij ILLER arriva à la conclusion qu'il est possible dans l'alimentation des vaches, de diminuer l'apport d'aliments grossiers, volumineux jusqu'à environ $2,5 \mathrm{~kg}$ par Ioo $\mathrm{kg}$ de poids de bête et que même on peut, pendant un temps assez court, supprimer complètement les aliments grossiers, volumineux. Avec une telle alimentation il n'y eut pas de troubles dans la santé des vaches et la production de lait ne fut pas troublée.

MüLLER considère d'ailleurs d'un autre point de vue les expériences au cours desquelles il fit varier l'apport d'aliments grossiers volumineux, et se demanda comment agissait une augmentation d'apport de cet aliment volumineux qui, dans ses expériences était de la paille de seigle. On peut résumer comme suit sa conclusion : "On ne peut pas considérer la paille de seigle comme un ballast néfaste, mais comme une partie directement utile de la ration ".

DAVENPORT (IO) fit d'intéréssantes expériences avec les veaux. Il essaya de les nourrir sans aliments grossiers volumineux. Il réussit à maintenir cette alimentation, dans un cas jusqu'à 5 mois, et, dans l'autre cas, jusqu'à 7 mois. La première bête qui avait été nourrie uniquement avec de l'avoine et du maïs dut être abattue car elle était complètement épuisée. L'autre, qui avait seulement bu du petit lait, reçut un aliment grossier volumineux dès qu'il parut dépérir ; il se remit alors complètement. Quelques heures après avoir reçu cet aliment grossier volumineux, il se mit pour la première fois dans sa vie à ruminer et se développa par la suite normalement.

Pour résumer ces différents résultats, nous pouvons dire que les différents auteurs sont d'accord pour estimer qu'une vache a besoin d'une ration qui lui donne le sentiment d'être rassasiée et que, par conséquent, il est indispensable d'établir des rations qui rassasient l'animal. 


\section{II. - De la nécessité d'éviter une surcharge de l'organisme digestif.}

Inversement il faut soigneusement éviter de surcharger l'organisme digestif de la vache. C'est la deuxième raison pour laquelle nous devons nous efforcer de connaître le volume convenable d'aliments à fournir dans une alimentation rationnelle.

LEROY (34) place cette considération en tête de sa théorie du "volume alimentaire " et l'exprime en disant qu'il ne suffit pas de fournir à l'animal les unités fourragères voules " mais qu'il faut aussi veiller à ce que les quantités d'aliments, estimées satisfaire les besoins nutritifs de l'animal, ne dépassent pas ses capacités digestives, et qu'il faut avant tout éviter de troubler la santé de l'animal par un excès d'aliments ".

Hansson (22) (page I33) a démontré qu'une ration trop volumineuse élève inutilement le travail à fournir par l'appareil digestif et trouble l'activité des organes respiratoires. C'est aussi la cause qui empêche l'absorption de trop fortes quantités d'aliments concentrés.

HoNcamp (25) (page I77) a examiné le côté économique de la nourriture et dit : "Il n'est pas économique de fournir à l'animal plus d'aliments grossiers volumineux qu'il n'est nécessaire pour le rassasier ».

HAGEMANN (I9 (page 249) dit que la grandeur de la ration à fournir aux bovins est très variable. La limite de cette grandeur est fixée par les capacités du système digestif que l'on ne doit pas surmener sans risquer de rendre la bête malade. Le praticien détermine cette limite d'après son expérience, qu'il a surtout acquise avec l'engraissement des boufs.

"Dans le cas d'engraissement des animaux il existe un régulateur normal qui détermine la quantité d'aliments absorbés, à savoir la faim qui pousse l'animal à manger jusqu'à ce qu'il soit rassasié, ce qu'il ne fait du restele plus souvent que d'une manière peu convenable. Au cours de la période de nourriture intensive fournie au cours de la période d'engraissement, les bêtes se sont habituées à remplir le plus complètement possible leur estomac et leur système digestif. $\mathrm{Si}$ on leur fournit des aliments qui satisfont particulièrement leurs goûts elles ont tendance à avaler plus d'aliments, ce qui peut facilement amener à une suralimentation qui les empêchera de manger l'apport ou les apports suivants d'aliments ou amènera des troubles digestifs. Quoi qu'il en soit une telle alimentation trop forte amènera un recul de l'engraissement au lieu de le faire progresser ". Nous savons que ce principe ne s'applique pas seulement aux bêtes à l'engraissement, mais également aux vaches laitières dont la production en lait peut reculer fortement dans le cas de suralimentation.

Dans le cas des vaches déjà pleines, et approchant du vêlage, il faut également éviter la surcharge des organes digestifs, point sur lequel insiste M. FALCK (I4).

PoTT (44) souligne les dangers d'une alimentation trop volumineuse qu'il 
estime pouvoir amener un ralentissement de l'activité des muscles du système digestif et les affaiblir d'une manière définitive, ce qui - chez les ruminants cause des gonflements de la panse.

En Angleterre et en Amérique, on insiste énergiquement pour qu'il soit évité une trop forte surcharge des organes digestifs. Sous les auspices du Ministère Anglais de l'Agriculture, Boutruour (2) a établi des normes pour améliorer le rendement des vaches laitières grâce à une alimentation plus rationnelle. Puis Bucher (5) a souligné combien il était important de ne pas surcharger l'estomac des vaches. Il prohibe tout hachage des aliments et diminue la ration de racines au minimum, quand il ne la supprime pas totalement. Il estime que la contenance en ballast de la ration ne doit pas dépasser $3,9 \mathrm{~kg}$. D'après lui, on ne doit pas laisser les vaches manger ad libitum les aliments grossiers, volumineux comme cela est fait couramment, car non seulement on gâche ainsi ces aliments volumineux, mais on surcharge également l'estomac.

Les aliments contenus dans le système digestif de la vache remplissent rarement plus de $75 \%$ de son volume. Si les $25 \%$ de volume disponible sont remplis, il se forme une masse compacte ; le pétrissage nécessaire ne peut se faire, de sorte que l'aliment fourni en plus continue son chemin sans être digéré. Le cultivateur doit s'efforcer de rassasier sa vache et de lui permettre la production correcte de lait en lui fournissant le minimum d'aliments volumineux. Les faibles rendements en lait de certaines vaches résultent d'un trop fort remplissage de l'estomac de la vache. Une vache, qui, après avoir été renfourrée, se couche et grogne, a mal à l'estomac. Une grande partie de la paille fournie en supplément quitte - d'après BoutFlour - la vache dans le même état qu'elle est entrée dans la gueule, c'est-à-dire non digérée ; il vaudrait donc mieux que cette partie de la ration n'ait jamais été fournie à la vache. Par un trop fort apport d'aliments grossiers volumineux on perd plus de lait que si on en donnait insuffisamment.

Nous avons rapporté ces vues à cause de leur originalité. Eilles sont en forte opposition avec l'effort que nous faisons en Allemagne pour couvrir la plus grande partie de la ration nécessaire à la production laitière en utilisant les aliments produits à la ferme, lesquels sont surtout composés d'aliments grossiers volumineux, et d'aliments aqueux succulents. Les succès que nous avons obtenus dans cette voie prouvent que, malgré l'opinion contraire de certains Chercheurs étrangers, nous sommes sur la bonne voie. Une vache qui, après avoir mangé, se couche et grogne n'a pas nécessairement, d'après notre opinion, mal à l'estomac, mais nous avons plutôt tendance à croire que ce grognement confirme sa satisfaction.

DEICKE (II) rapporte qu'en Amérique les normes de MORRISSON ont trouvé dans la pratique une large application ; or ces normes indiquent un rapport déterminé à maintenir dans la ration entre les aliments grossiers volumineux et les aliments concentrés.

Une donnée plus précise est également fournie par l'indication de la teneur 
en matière sèche de la ration. Si une ration contient une quantité suffisante de substances nutritives, mais trop de substances sèches, son action perd de son efficacité " parce que l'estomac de la vache est surchargé par cet aliment volumineux et que sa capacité d'absorption ne suffira pas pour absorber les grandes quantités d'aliments concentrés qu'il est nécessaire de faire avaler à la vache si on veut qu'elle produise de grosses quantités de lait. On a rassasié trop tôt la vache avant qu'elle ait eu le temps d'absorber les éléments nutritifs qu'il est nécessaire de lui fournir. Pour cette raison, on ne donne pas de paille, de menuepaille et de fourrage sec parce que de tels aliments absorbent une trop grande partie de la capacité d'absorption du système digestif sans apporter suffisamment d'éléments nutritifs ".

\section{III. - De la nécessité de fournir suffisamment d'éléments nutritifs, tout en rassasiant la vache.}

Les dernières idées que nous venons de citer font apparaître un troisième point très important : pour réaliser une alimentation rationnelle, il faut donner une nourriture qui rassasie la vache, mais qui - en même temps --- fournisse à celle-ci les éléments nutritifs dont elle a besoin. Nous avons souligné que la ration fournie doit donner à la vache le sentiment d'être rassasiée, sans que l'on surcharge son estomac, mais la vache doit également être en mesure d'avaler la ration que nous avons présumé devoir lui fournir les substances nutritives nécessaires à son entretien et à sa production.

SETTEGAST (48) examine dans son ouvrage cette question, et déclare que l'animal ne peut pas absorber des quantités suffisantes de substances nutritives, si on augmente trop dans la ration " les aliments occupant beaucoup de volume, ou trop riches en ballast $)$.

Hannsson (22) (page 8o) étudie cette idée en partant de la valeur alimentaire de la paille. Il considère qu'on peut l'employer comme aliment de remplissage, mais qu'il faut éviter d'en donner trop. "En effet, vu la faible valeur nutritive de la paille et ses caractéristiques d'encombrement (forte proportion de matière sèche par unité fourragère), on risque en donnant de fortes quantités de paille, d'empêcher l'animal d'avaler les quantités nécessaires d'aliment concentré ».

Comme Svoboda (5I) l'indique, nous devons bien distinguer "le rassasiement physiologique pa les substances nutritives " et le rassasiement volumétrique par le ballast ", ce dernier pouvant être considéré également comme un "rassasiement mécanique ». Nous devons exiger d'une ration adéquate qu'elle réalise en même temps le rassasiement physiologique et le rassasiement mécanique. Svoboda étudia le problème des étables de nourrisseurs où les vaches, en plus de la ration d'entretien et de production, doivent recevoir une ration d'engraissement. Là encore, comme dans le cas de la nourriture des vaches très grandes laitières, on risque d'obtenir le rassasiement mécanique avant d'avoir atteint le rassasiement physiologique. 
WI'TT (56) exprime la même pensée.

BüNGER (7) dit : “Une alimentation est irrationnelle si on atteint le rassasiement par les matières de remplissage ou ballast de 1'aliment sans avoir couvert en même temps les besoins en substances nutritives de l'animal, ou que, inversement, quand le corps a reçu les substances nutritives nécessaires, l'animal n'est pas en même temps rassasié. Fin d'autres termes il faut mener l'alimentation de manière à ce qu'au rassasiement par le ballast corresponde en même temps un rassasiement physiologique, c'est-à-dire qu'il ait été fourni à l'animal les substances nutritives dont il a besoin ".

WERNER (55), caractérise nos possibilités actuelles d'alimentation quand il prend position sur le problème de la manière suivante (page 55) : "Il est facile de bâtir une théorie de l'alimentation animale dans laquelle nous supposons avoir à notre disposition des aliments avec des valeurs extrêmes, c'est-à-dire très pauvres en ballast et très riches en protéines ou hydrocarbones. Mais nous devons compter à l'avenir avoir surtout à notre disposition des aliments avec valeurs moyennes, que ce soit pour la richesse en substances nutritives ou comme coefficient de digestibilité "..

Il faut aujourd'hui avec du fourrage, de l'ensilage et des betteraves fournir à l'animal les substances nutritives dont il a besoin pour son entretien et la plus forte production possible de lait. Il pourrait, par exemple, arriver que pour une vache produisant $20 \mathrm{~kg}$ de lait, on calcule une ration, et que l'on trouve que $25 \mathrm{~kg}$ de foin de prairie de qualité extra fournissent les substances nutritives nécessaires. La question se pose immédiatement de savoir si l'animal pourra absorber cette quantité de foin. Cette absorption ne sera-t-elle pas arrêtée parce que la vache aura sa faim rassasiée avant d'avoir mangé le total des $25 \mathrm{~kg}$ ? C'est là le grand art du nourrisseur de la vache : il doit savoir choisir avec compétence les aliments différents composant la ration, de manière à ce que le volume de ces aliments concorde avec ce qu'il faut fournir à la vache pour la rassasier, et, en même temps satisfaire aux besoins en substances nutritives résultant de la production de la vache.

Pour réaliser cette alimentation rationnelle, le nourrisseur habile doit connaitre toutes les caractéristiques de ses aliments et les besoins en substances nutritives de ses animaux. Il sera aidé par les tables lui indiquant les normes des aliments et les besoins des animaux. En général, les richesses en substances nutritives de nos aliments sont résumées par deux chiffres : la protéine digestible et la valeur amidon. Mais tout ce que nous venons de dire prouve qu'il est nécessaire que nous possédions un troisième chiffre qui nous permette de juger si l'organisme a reçu les éléments qui donnent à l'animal le sentiment d'être rassasié, ou inversement qu'il n'est pas fourni plus d'aliments que l'organisme n'en peut absorber. 


\section{C. - ELEMENTS PERMETTANT DE MESURER L'ACTION RASSASIANTE D'UN ALIMENT ET LES BESOINS DE LA VACHE POUR ETRE RAS- SASIEE}

Nous allons examiner comment cette question a été traitée dans la littérature sur l'alimentation animale.

\section{I. - Les quantités absolues d'aliments :}

Le premier criterium de l'action rassasiante d'un aliment fut d'abord la " valeur fourrage ". ThaER (53) au paragraphe 48 de ses Principes d'une A griculture Rationnelle dit : "La ration convenable d'une vache adulte de taille moyenne paraît être de 18 livres de foin dont la moitié peut être du reste avantageusement remplacée par des racines : une bonne ration peut également être de 80 livres de trèfle vert. De grosses vaches peuvent recevoir 25 à 30 livres d'aliment sec et II 2 à I 40 livres d'aliment vert ; on peut en outre leur donner autant de paille qu'elles peuvent en manger \%. Les besoins en éléments nutritifs et le rassasiement de la vache paraissent ainsi être définis clairement. Quoique nous ayons le droit de considérer que les indications sur les besoins exacts en substances nutritives sont très insuffisants, nous ne devons pas oublier l'état d'avancement des connaissances à l'époque. WERNER (55) dit à ce sujet : "A l'époque de ThAER, on utilisait surtout des aliments grossiers volumineux. Les proportions assez faibles d'aliments aqueux succulents et d'aliments concentrés employés n'influençaient guère le volume de la ration. Il en résultait, au point de vue volume de la ration quotidienne, une compensation interne qui excluait les problèmes que nous rencontrons aujourd'hui... "

Comme successeurs de THAER dans la théorie de la "valeur fourrage " nous citerons les noms de Block-KAPpe, PABst et Schweiger (voir MANgold, 37). Cette théorie de la "valeur fourrage " a longtemps été appliquée par les savants et les agriculteurs (voir Werner, 55, page 53). Ceci s'explique par le fait que cette notion de "valeur fourrage " était particulièrement simple et que l'on satisfaisait à peu près aux exigences d'une époque, qui ne se souciait pas encore trop des questions de substances nutritives nécessaires et de rassasiement. Par la suite, on estima en général que le rassasiement dépendait de la quantité pure et simple d'aliments fournis quoiqu'à cette époque les Chercheurs avaient déjà reconnu que l'action nutritive d'un élément dépendait de sa teneur en certaines substances nutritives. On peut citer le nom de V. GoHren (I7) qui peut-être le premier (voir BrüGGEMANN (4), page 298) parle du rapport entre le volume des organes digestifs et distingue soigneusement entre les aliments concentrés et volumineux. V. GOHREN donne les quantités absolues d'aliments grossiers volumineux tels que le fourrage qui peuvent être absorbés par les différents animaux (chevaux, bovins et moutons). D'après lui, une vache a besoin 
de 6 à 30 livres d'aliments grossiers volumineux pour être rassasiée. V. GOHREN se refuse à considérer tout autre critérium de l'action rassasiante, tel que la teneur en matière sèche quoiqu'il admet que le volume peut être " réglé et représenté " par les quantités de matière sèche.

Boussignault (3) (voir WFRner (55) page 54) a essayé d'envisager la théorie de la " valeur fourrage ", non seulement du point de vue des éléments nutritifs mais aussi du point de vue du volume. I1 divisa les aliments en deux catégories : dans la première se trouvaient les aliments concentrés et dans la deuxième les aliments grossiers volumineux, pour chacun desquels il indiquait combien de $\mathrm{kg}$ de paille correspondaient pour apporter la "valeur fourrage " équivalente.

SETTEGAST (48) fit un nouveau pas en avant presqu'à la même époque que V. Gohres. Il utilise la quantité absolue d'aliments grossiers volumineux donnés comme mesure du volume nécessaire et absorbable (page 403). Il estima que les limites possibles d'apport quotidien d'aliments volumineux étaient de 6 à 30 livres. Mais déjà SETTEGAST conseille d'utiliser la matière sèche comme élément de mesure de la quantité d'aliment grossier volumineux qu'il est possible de fournir (page 402). Il justifie l'emploi de la quantité d'aliments grossiers volumineux comme mesure de l'action rassasiante en disant : "Les mêmes quantités de substances nutritives, y compris les fibres de la plante des différents aliments occupent à peu près le même espace dès qu'elles sont englobées dans le bol alimentaire; de plus, c'est la partie non digestible des fibres boisées des aliments volumineux qui surtout déterminent le ballast. Nous considérons donc que nous pouvons exprimer le volume alimentaire de l'aliment simplement par le poids d'aliments grossiers volumineux à fournir à l'animal ".

\section{II. - La teneur en matière sèche des aliments :}

SETTEGast arrive donc déjà à l'idée que la teneur en matière sèche d'un aliment est un critérium de son action rassasiante.

Grouven (I8), qui publia ses premières tables de normes alimentaires en 1860, y indiquait déjà les quantités de matière sèche nécessaires pour des animaux de différentes espèces de différents poids.

HENNEBERG (24) entreprit vers I86o six séries de techerches sur la ration d'entretien des bœufs. Il compléta la théorie de la " valeur fourrage " en introduisant la conception des substances nutritives, et d'après WERNER (55) plaça le problème du rassasiement de l'animal sur un plan nouveau.

Par la suite, nous trouvons dans tous les ouvrages, tables et travaux scientifiques la masse de matière sèche comme élément de mesure de l'action rassasiante d'un aliment. C'est sur cette base que les savants des questions alimentaires fondent leurs théories et leurs idées, dont nous allons exposer maintenant les principales.

KüHN (30) estime recommandable un apport de substance sèche de I 8 à 
$33,5 \mathrm{~kg}$ par I ooo $\mathrm{kg}$ de poids vif. D’après lui (page I3I) il ne faut pas en général fournir une quantité d'aliments grossiers volumineux tels que fourrage et paille inférieure au $\mathrm{r} / \mathrm{I}$ ooo du poids vif, soit Io $\mathrm{kg}$ dans le cas où on y est obligé on peut descendre au minimum à 6 ou $8 \mathrm{~kg}$ par I ooo $\mathrm{kg}$ de poids vif. Dans les conditions normales il est recommandé de donner le $\mathrm{I} / 5^{\circ}$ ou $\mathrm{I} / 8 \mathrm{o}$ du poids vif sous forme d'aliment grossier volumineux.

Les chiffres les plus utilisés aujourd'hui sont ceux de KELLNER (26). Celuici indique pour une vache à lait de $\mathrm{I}$ ooo $\mathrm{kg}$ de poids vif les chiffres suivants d'apport quotidien de matière sèche :

\begin{tabular}{l|c|c|c|c|c}
\hline \hline & Entretien & \multicolumn{3}{|c}{ Production quotidienne de lait } \\
\cline { 2 - 4 } & $5 \mathrm{~kg}$ & $10 \mathrm{~kg}$ & $15 \mathrm{~kg}$ & $20 \mathrm{~kg}$ \\
\hline $\begin{array}{c}\text { Kg de matière sèche dans l'aliment } \\
\text { fourni........................... }\end{array}$ & $15-21$ & $22-27$ & $25-29$ & $27-33$ & $27-34$ \\
\hline
\end{tabular}

KELLNER admet que l'on peut dans la pratique s'écarter de Io \% de ces chiffres. Du reste, d'après lui, ces écarts n'ont pas grande importance car les bêtes s'habituent au changement de volume de l'aliment (page 407). KELLNER estime que le besoin en matière sèche, en fonction de la production du lait ne croît pas suivant une règle linéaire, mais beaucoup plus lentement vu les possibilités d'absorption limitée du système digestif.

Svoboda (5I) complète les données de KELLNER en observant que les besoins en matière sèche des animaux de petite taille sont relativement plus élevés. Il détermine un système dans lequel la matière sèche à fournir est calculée en fonction du poids vif et de la production de lait de l'animal. Il indique les chiffres suivants :

\begin{tabular}{|c|c|c|c|c|}
\hline \multirow{2}{*}{ Poids vif } & \multicolumn{4}{|c|}{ Matière sèche (kg) à fournir pour une production quotidienne de lait de } \\
\hline & $5 \mathrm{~kg}$ & to $\mathrm{kg}$ & $15 \mathrm{~kg}$ & $20 \mathrm{kgr}$ et plus \\
\hline $35^{\circ}$ & 10,5 & I I, 7 & I 3,2 & I 3,5 \\
\hline 400 & $\mathrm{I} I, \mathrm{~T}$ & I 2,3 & T 3,8 & I 4,5 \\
\hline $45^{\circ}$ & I I, 7 & 12,0 & 14,4 & $\mathrm{I} 4,7$ \\
\hline 500 & I 2,3 & I 3,5 & I 5,0 & I 5,3 \\
\hline 550 & I 2,8 & $\mathrm{I}_{4,1}$ & I 5,6 & 15,8 \\
\hline 600 & I 3,3 & $I_{4}, 6$ & $I 6,1$ & I 6,3 \\
\hline 650 & i 3,9 & $\mathrm{I}_{5}, \mathrm{I}$ & I 6,6 & 16,9 \\
\hline 700 & {$[4,4$} & I 5.6 & 17,1 & I 7,4 \\
\hline $75^{\circ}$ & 14,0 & 16,1 & 17,6 & 17.9 \\
\hline
\end{tabular}

Svoboda déclare que ces normes permettent un rassasiement physiologique et mécanique de la vache.

Muray (4I) est également d'avis que c'est la teneur en matière sèche de 
1'aliment qui détermine le rassasiement du bovin, mais souligne les grands écarts existant entre les chiffres donnés à ce sujet par les différents auteurs. Il rapproche les chiffres suivants indiqués par différents auteurs pour I $000 \mathrm{~kg}$ de poids vif.

Besoins en matière sèche pour I ooo kg de poids vif pour les vaches laitières.

\begin{tabular}{|c|c|c|c|}
\hline D'après & D'après & D'après & D'après \\
\hline WOLFF- & KELLNER & MORRISSON & ARMSBY \\
\hline LEHMANN & & & \\
\hline $25-32 \mathrm{~kg}$ & $22-34 \mathrm{~kg}$ & $26 \mathrm{~kg}$ & $20-30 \mathrm{~kg}$ \\
\hline
\end{tabular}

Comme Muray, WOOD repousse la conception d'après laquelle les besoins en matière sèche d'un animal varient proportionnellement à son poids vif et qu'il soit possible par un simple calcul de déduire des besoins en matière sèche pour I ooo kg de poids vif les besoins en matière sèche pour des poids vifs différents. Muray conclut de ses recherches que la capacité d'absorption des aliments et le poids vif ne varient pas proportionnellement et que la méthode habituelle de calcul en partant des besoins en matière sèche pour I ooo $\mathrm{kg}$ de poids vif est fausse.

Les normies de MORRISson (voir DEICKE, II) qui sont très appliquées en Amérique, indiquent un certain rapport entre l'aliment grossier volumineux et l'aliment concentré. La recommandation d'une certaine teneur en matière sèche dans la ration doit donc permettre une mesure suffisamment précise de la grandeur de la ration.

Nous trouvons également chez beaucoup de savants la notion que c'est la teneur en matière sèche qui détermine l'action rassasiante d'un aliment et les possibilités d'absorption de la ration par la vache. Citons PUTt (44), HoNCAMP (25), HANSEN (20, 2I), H. FALCK (I4, I5), KRONAChER (29) et SchmidT (46).

\section{III. - Elément établissant une relation entre les différents éléments constitutifis d'un aliment :}

Différents chercheurs ont essayé de solutionner la question du volume de la ration en rapprochant sa valeur nutritive de sa teneur en matière sèche et de déterminer ainsi la possibilité d'un aliment à remplir le système digestif et à rassasier l'animal.

Le chercheur français LEROY (34) a proposé de calculer le coefficient d'encombrement des aliments. Ce coefficient est le rapport de la matière sèche relativement à la valeur alimentaire. LEROY a établi des tables où ce coefficient est indiqué. Il part de ce fait que l'herbe a le coefficient le plus favorable à savoir I,3. Aussi, doit-on s'efforcer dans nos rations d'obtenir ce coefficient de I,3. Voyons un exemple de calcul suivant cette méthode :

Une vache pesant $700 \mathrm{~kg}$ et produisant $30 \mathrm{~kg}$ de lait a besoin de $\mathbf{I}_{5}, 03 \mathrm{uni}$ - 
tés fourragères contenant $2 \mathrm{I}, 89 \mathrm{~kg}$ de substance sèche. Le coefficient volumétrique est dans ce cas de :

$$
\frac{\text { matière sèche }}{\text { unités fourragères }}=\frac{21,89}{\mathrm{I} 5,03}=1,46
$$

LEROY mentionne que l'appétit de la vache ne diminue pas ou que la valeur alimentaire de la ration n'est pas défavorablement influencée, quand on remplace dans cette ration une partie des aliments concentrés par du foin ou de la paille. On peut ainsi changer la teneur en matière sèche de la ration sans influencer sa valeur alimentaire.

Hansson (22) qui utilise également la matière sèche comme base pour déterminer l'action rassasiante d'un aliment et les besoins d'une vache pour avoir son appétit rassasié, calcule le degré de concentration d'un aliment, de manière à faire apparaître la capacité de cet aliment à rassasier un animal. I1 indique (page I33) que la concentration en éléments nutritifs d'un aliment doit subir des variations considérables suivant la production de lait de la vache. Hansson calcule la concentration en éléments nutritifs d'après la formule suivante :

$$
\frac{\text { matière sèche }}{\text { unités fourragères }}=\frac{\text { IOO }}{\mathrm{u}} \text {. }
$$

Avec la paille de seigle on trouve avec cette formule un degré de concentration de 24 ; pour du fourrage de trèfle 49 , et pour du tourteau d'arachide I4o. Avec des vaches sèches un degré de concentration de 45 suffit, ce qui signifie qu'on peut les nourrir avec de la paille et du fourrage. Au contraire, avec des vaches donnant de fortes productions de lait, il faut un degré de concentration de 7 o.

BuNger (7) définit la " concentration en ballast " qui est le rapport :

\section{Ballast \\ Matières nutritives totales}

Il estime, par exemple, important dans la nourriture des volailles de connaitre la concentration en ballast la plus convenable et quel volume l'ensemble de l'aliment doit avoir pour une production déterminée ".

Comme Leroy (34), Hansson (22) et Bünger (7), Werner (54) estime que la concentration en éléments nutritifs dans une ration doit être plus forte, quand la production de l'animal est plus élevée. La digestibilité de la ration doit varier avec l'importance de la production de lait.

\section{IV. - La teneur en ballast des aliments :}

Beaucoup de savants ont considéré que c'était le ballast qui était 1'élément permettant de déterminer l'action rassasiante des aliments et les besoins de la vache pour être rassasiée. La théorie du ballast est en fait la dernière étape de l'évolution des idées sur le rassasiement de l'animal. Nous trouvons déjà l'indi- 
cation du ballast dans les travaux de SETTTEGAST (47) publiés en I 868 et qui ont déjà été mentionnés dans cet article. Nous croyons bon de reproduire ici le passage principal (page 376) de SETTEGAST sur ce sujet :

"La partie non digestible ou non digérée, qui échappe dans le système digestif aux processus de dissolution et transformation, est le ballast, lequel est rejeté du corps sous forme d'excréments. Le ballast n'est pas directement nécessaire pour la nourriture de l'animal, mais il sert à remplir les organes digestifs, et est particulièrement important dans le cas des ruminants".

Pour mesurer l'action rassasiante d'un aliment SETTEGAST n'utilise cependans pas la teneur en ballast de l'aliment, mais soit le poids brut de cet aliment, soit la quantité de matière sèche qu'il contient.

C'est surtout LEHMANN qui a développé la conception d'un ballast en tant qu'élément déterminant l'action rassasiante d'un aliment et les besoins de l'animal pour être rassasié. L,EHMANn n'entreprit pas lui-même de recherches et établit sa théorie et ses chiffres d'après les données résultant des recherches de WOLFF. Il en déduisit que la quantité de ballast absorbée par une vache est un chiffre constant égal à $4,3 \mathrm{~kg}$.

Cette théorie est basée sur l'hypothèse que ce sont seulement les parties organiques non digestibles de l'aliment et non absorbées par le système circulatoire grâce à l'action des sucs digestifs qui déterminent le sentiment de rassasiement chez l'animal. La teneur de l'aliment en éléments organiques digestibles doit donc être sans influence sur le rassasiement de l'animal, car ces substances ne tardent pas à quitter le système digestif et par suite n'exercent plus aucune pression sur les organes du système digestif. Il est également estimé que les parties non organiques de 1'aliment exercent une action négligeable au point de vue rassasiement.

Le chiffre moyen de $4,3 \mathrm{~kg}$ de ballast pour une vache de $500 \mathrm{~kg}$ est calculé par LEHMANN (33) de la manière suivante:

" Un bovin de $500 \mathrm{~kg}$ de poids vif a besoin pour son entretien d'une ration qui contient : $8,8 \mathrm{~kg}$ de substance organique dont $4,5 \mathrm{~kg}$ sont digestibles, soit $4,3 \mathrm{~kg}$ de ballast ou substances organiques non digestibles. Le même calcul indique pour une vache de $500 \mathrm{~kg}$ donnant 8 à $\mathrm{Io} \mathrm{kg}$ de lait un besoin de $\mathrm{r} 2 \mathrm{~kg}$ de substance organique dont $7,7 \mathrm{~kg}$ sont digestibles, soit $4,3 \mathrm{~kg}$ de ballast. "

En ce qui concerne ce chiffre de $4,3 \mathrm{~kg} \mathrm{~L}_{\mathbf{H}}$ HMANN (32) explique également:

"Ce chiffre n'est pas toujours égal pour tous les animaux car la capacité d'absorption de ballast peut diminuer lorsque les animaux sont élevés avec des aliments pauvres en ballast. Quand l'animal est devenu adulte, ce chiffre ne change plus guère, tout au moins d'une manière sensible. Quand une vache reçoit pour son entretien, c'est-à-dire pour maintenir son poids, du fourrage et de la paille, elle reçoit 3 unités amidon et $4,3 \mathrm{~kg}$ de ballast. Si cette même vache produit 20,30 ou $40 \mathrm{~kg}$ de lait, la quantité de ballast absorbée par elle ne change pas mais elle doit recevoir une quantité plus forte d'éléments nutritifs. "

LEHMANN affirme donc que " le corps de chaque animal adulte est pour 
ainsi dire étalonné pour recevoir une quantité déterminée « de ballast ». La quantité maxima possible d'aliment que peut absorber l'animal est donc déterminée par la partie organique non digestible de la ration. LEHMANN en déduit que les aliments donnés à une vache doivent être d'autant plus digestibles que la production en lait de la vache est plus élevée. Cette question doit être particulièrement considérée quand on examine si la ration qu'on a calculée peut être absorbée par l'animal. LEHMANN indiqua pour une vache de $500 \mathrm{~kg}$ les normes suivantes :

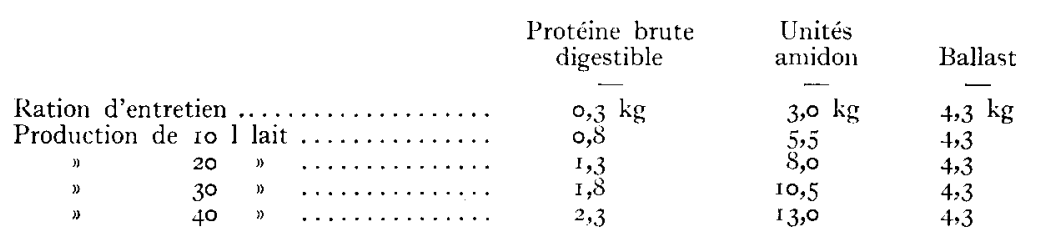

Ce chiffre de $4,3 \mathrm{~kg}$ de ballast depuis qu'il a été déterminé par le Pr LEHMANN a été très étudié dans toutes les théories sur l'alimentation. Nous trouvons le chiffre mentionné dans le calendrier de Catermann, dans l'ouvrage de WERNER (54), dans les tables du Reichsnährstand établies par KIRSCH et WERNER, et dans les tables simplifiées de BüNGER (9).

Mais il ne manque pas de savants qui expriment des doutes sur la théorie du ballast de Lemmann. On rappela d'abord que d'éminents spécialistes de l'alimentation ne s'étaient pas servis dans leurs ouvrages de la notion de ballast, c'est ce que fit FINGERI ING qui avait toujours utilisé la matière sèche comme élément déterminant le rassasiement. Schmid (46) fait comme Fingeri,ıng ; il dit (page I29) que dans une ration c'est la teneur totale en matière sèche qu'il faut surtout considérer, "cette teneur ayant du reste une certaine relation avec le besoin en ballast de l'animal ".

HANSEN (20) fait une critique détaillée de la théorie du ballast de LEHMANN. Il estime que LEHMANN préfère calculer le ballast car il sépare ainsi mathématiquement la partie digestible de la partie non digestible de la ration, alors que le calcul de la matière sèche englobe les deux parties. HANSEN conclut :

"Finalement, les deux méthodes de calcul aboutissent au même résultat. Si une vache reçoit autant de matière sèche qui lui est nécessaire ou le maximum qu'elle peut absorber, les quantités de substances nutritives nécessaires à 1'entretien et à la production seront présentes dans cette matière sèche et par conséquent nous aurons satisfait à toutes les exigences. "

MEINHOLD (38) doute de la constance du chiffre du ballast indiqué par LEHMANN et entreprit des recherches sur la question. Il en déduisit les conclusions suivantes :

"L'augmentation de la production du lait après le vêlage cause une augmentation d'absorption dé la matière sèche (substance digestible et non digestible) ainsi que du ballast (substance non digestible). Puis quand la production du lait diminue, cette absorption décroît également. " . 
Sur une question de ma part, Meinhold m'a communiqué les chiffres cidessous pour une vache de $550-600 \mathrm{~kg}$ de poids vif :

\begin{tabular}{cc||cc}
$\begin{array}{c}\text { Production de lait } \\
(\mathrm{kg})\end{array}$ & $\begin{array}{c}\text { Ballast } \\
(\mathrm{kg})\end{array}$ & $\begin{array}{c}\text { Production de lait } \\
(\mathrm{kg})\end{array}$ & $\begin{array}{c}\text { Ballast } \\
(\mathrm{kg})\end{array}$ \\
- & - & - & - \\
$0-5$ & $3,20-3,60$ & $15-20$ & $4,20-4,50$ \\
$5-10$ & $3,70-4,00$ & $20-25$ & $4,40-5,00$ \\
TO-15 & $3,90-4,30$ & $25-30$ & $4,90-5,50$
\end{tabular}

MEINhol, estime que ces chiffres, calculés d'après ses essais pratiques, montrent que la quantité de ballast n'est pas une constante, mais croît avec la production de lait. Il estime que les quantités les plus faibles de matière sèche et de ballast nécessaires au rassasiement de la vache sont absorbées 8 à I 2 semaines avant le vêlage. Au contraire, au cours des dernières semaines précédant immédiatement le vêlage on peut constater non seulement une augmentation de besoins en substances nutritives mais aussi une augmentation en besoins de matière sèche et ballast. Ces observations contredisent donc 1'opinion assez courante que dans les dernières semaines précédant le vêlage la capaciré d'absorption d'aliments par la vache est diminuée, parce que le volume des cavités abdominales est diminué par la place prise par le fotus. Enfin, toutes les observations de Mrinhold sont en contradiction avec la théorie de LEHMANN sut la constance $d u$ ballast. Une remarque de MoLLGAARD (40) (page I 8 I) à ce sujet est importante. Discutant le résultat de ses recherches avec des vaches non pleines, qui d'abord, donnaient de fortes quantités de lait puis devenaient ensuite sèches, il mentionne "les difficultés qui résultèrent de ce que les vaches sèches ne voulaient généralement pas absorber les grosses quantités d'aliment qui leur avaient été fournies et qu'elles absorbaient alors qu'elles étaient en lactation, en particulier celles fournies quand leur production de lait était élevée ». A un autre endroit (page I47), MolLGAARD écrit que les vaches très grosses productrices de lait $(20-30 \mathrm{~kg}$ de lait) ne peuvent pas généralement complètement digérer au début de la lactation des quantités suffisamment fortes d'aliments, et que c'est seulement au bout de 2 à 3 semaines qu'elles s'y habituent.

Meinhold (38) discute la question de savoir si c'est la teneur en matière sèche ou en ballast qui doit être prise comme base pour juger de l'action rassasiante d'un aliment. Il donne de nombreux exemples qui prouvent qu'il existe de grosses différences entre les quantités de matière sèche absorbée, lesquelles résultent des différences de digestibilité et en particulier des teneurs différentes en fibres des aliments. Les différents auteurs ont indiqué des variations importantes de la matière sèche absorbée :

$$
\text { KELLNER : } 22-34 \mathrm{~kg} \text { HANSEN : } 15-35 \mathrm{~kg} \quad \text { FALCK }: 20-30 \mathrm{~kg}
$$

MEINHOLD compare ces variations de matière sèche avec les variations qu'il a trouvées dans ses expériences pratiques pour le ballast. Il en conclut que le ballast fournit une base beaucoup plus sûre pour calculer la capacité d'absorption et les besoins des animaux pour être rassasiés, car les variations du ballast 
sont relativement moins fortes que celles de matière sèche. Si on fournit à 1'animal une quantité déterminée de matière sèche sous forme d'aliment riche en fibres et par conséquent en ballast (par exemple des pailles), la valeur de matière sèche amenant le rassasiement est tout à fait différente de celle dans le cas où on fournirait la même qiuantité de matière sèche sous forme d'aliments très digestibles et pauvres en ballast.

Meinhold souligne que dans le cas des vaches au pâturage la question du ballast joue un rôle énorme car les vaches mangent jusqu'à avoir leur besoin en ballast satisfait et non jusqu'à rassasiement physiologique. Il est du reste très probable qu'il se produit dans ce cas un gaspillage d'éléments nutritifs.

BUCHER (5) attache une grosse importance à l'application de la théorie du ballast pour réaliser une alimentation rationnelle. Il rappelle (page 6o) que le $D^{r}$ GLÄTTLI est arrivé à la conclusion que $40 \mathrm{~kg}$ d'herbe de pâture fournissent à une vache non seulement sa ration d'entretien, mais lui permettent également de produire 6 à $9 \mathrm{~kg}$. de lait. Comme dans cette quantité d'aliments il ne se trouve que I,24 kg de ballast, ce qui est loin de la constante de 4,3 kg il doute qu'on puisse maintenir la théorie de la constance du ballast. Comme on est en droit de douter qu'une vache de $500 \mathrm{~kg}$ puisse jamais réussir à manger les I50 kg d'herbe fraîche qui la rassasieraient, et si on admet en outre qu'il faur $4,3 \mathrm{~kg}$ de ballast pour la rassasier, il semble logique de conclure, que le chiffre de $4,3 \mathrm{~kg}$ doit être considéré comme une donnée maxima. WIEGNER, peu de temps avant sa mort, déclarait estimer que ce maximum devait même être diminué.

BUCHER a finalement exprimé 1'opinion que "la capacité d'absorption de la vache dépend non seulement du poids de la partie non digestible des substances organiques nutritives, mais aussi de l'espace occupé par l'ensemble des parties digestibles et non digestibles $)$.

BüNGER (8) souligne la différence entre la teneur en ballast d'un aliment et le volume que l'aliment occupe. Les actions dues au ballast et au volume agissent dans la même direction, mais la différence entre le rassasiement par le ballast et le rassasiement par le volume réside dans le fait que le rassasiement par le ballast a une durée plus longue que le rassasiement par le volume, car l'eau contenue dans les aliments volumineux (pommes de terre, betteraves, ensilage) est rapidement résorbée et laisse de la place libre pour une nouvelle absorption d'aliments.

WiTT $(56)$ a également disséqué la théorie du ballast de LEHMANN dont nous indiquerons les conclusions particulièrement intéressantes. WITT fait l'hypothèse que le chiffre du ballast absorbé est une constante pour chaque vache. Pendant l'alimentation d'hiver on détermine la quantité de ballast que chaque vache peut absorber, et on en déduit la quantité d'herbe que la vache peut manger pendant 1'été. D'après lui, si on calcule les rendements possibles pour une vache à l'herbage en se contentant de tenir compte des substances nutritives absorbées nécessaires pour l'entretien et la production, on aboutit toujours à des erreurs, car la vache ne mange pas pour satisfaire son appétit 
physiologique, mais jusqu'à ce qu'elle soit rassasiée mécaniquement, c'est-à-dire ait absorbé la quantité de ballast qui la rassasie.

WITT $\left(5^{8}\right)$ indique également que souvent on cherche à donner à une vache, qui est déjà rassasiée en ballast, un supplément de nourriture pour lui faire absorber plus de substances nutritives. Dans ce cas, comme la vache ne peut absorber une quantité de ballast supérieure à celle qui la rassasie, elle refuse de manger ce supplément, ou elle laisse sans la manger une partie de la ration rassasiante de base. D'après WITT, ce fait devrait être considéré de plus près quand on établit des rations : les aliments concentrés contiennent aussi du ballast. Il peut en résulter qu'une vache à laquelle on donne des aliments concentrés laissera sans la manger une partie de la ration de base, partie dont le ballast correspond au ballast apporté par l'aliment concentré. Il en résulte que le fait de donner des aliments concentrés diminuera l'apport de substances nutritives par la ration de base. "Le supplément d'aliment concentré n'apportera pas forcément à la ration totale un supplément de substances nutritives correspondant exactement auxdites substances, contenues dans l'aliment concentré ; ce supplément d'aliment concentré ajoute à la ration totale de sa "valeur de remplissage". L'auteur donne alors des exemples de cette "valeur de remplissage " pour de nombreux aliments.

Il en ressort alors des particularités intéressantes, quand, partant de ce point de vue, on examine l'action d'un apport d'aliments complémentaires (aliments concentrés, pulpes séchées, etc...) aux vaches qui pâturent. Il en résulte qu'il y a alors simple échange, l'aliment concentré prenant la place de l'herbe, et finalement on n'obtient pas forcément une augmentation des substances nutritives absorbées mais il arrive au contraire que le total de ces substances nutritives absorbées diminue et que finalement la production baisse.

On doit, dans la pratique, tenir compte des déductions de WiTr. Malgré tout, il n'a pas prouvé que la quantité de ballast absorbé est une constante. Non seulement, nous manquons de recherches précises à ce sujet, mais cette hypothèse, comme nous l'avons montré est discutée.

De toutes ces considérations, on peut déduire combien il est important de connaître les besoins de la vache pour être rassasiée et l'action rassasiante des aliments. Les principales unités utilisées sont :

Io Le poids brut des aliments;

$2^{\circ}$ La teneur en matière sèche des aliments ;

$3^{\circ}$ Une unité qui fait apparaître un rapport entre différents éléments constitutifs de l'aliment ;

$4^{\circ}$ La teneur en ballast des aliments.

Or, aucune de ces unités n'a pu se révéler d'une application certaine et on ignore encore aujourd'hui quelle unité nous permet de juger du rassasiement de l'animal.

Les travaux récemment publiés par BRügGEMANN (4) confirment ce point de vue. Ses recherches sur des bœufs en croissance n'ont pu apporter aucune 
conclusion sur l'unité qui permettrait de juger de manière précise l'action rassasiante d'un aliment. Finalement, après avoir indiqué les volumes d'aliment qu'il lui paraissait convenable de donner pour le but à atteindre, il note (page 374) les teneurs en matière sèche et ballast des différents aliments. Puis il laisse à l'éleveur la liberté de déterminer le choix entre les deux chiffres, ou l'emploi simultané de ces deux chiffres pour fournir aux bœufs la quantité d'aliments qui les rassasiera.

On peut même admettre que LEHManN lui-même n'était pas complètement convaincu de sa théorie du ballast. Cela semble ressortir d'une de ses remarques au Congrès International Avicole de Leipzig en I936, au cours de la discussion sur la Conférence de BüNGER (8). Expliquant comment il était arrivé au chiffre de $4,3 \mathrm{~kg}$ de ballast pour un animal de $500 \mathrm{~kg}$ de poids, il déclara: " J'ai choisi comme ballast la quantité de substances organiques non digestibles. Il aurait peut-être été préférable de choisir la matière sèche non digestible ; mais ceci n'était pas possible car nous ne possédions pas les chiffres nécessaires, alors que nous avions tous les éléments voulus pour la substance organique. "

Nous trouvons une remarque analogue chez Wrтt (56) qui compare la valeur rassasiante de ro $\mathrm{kg}$ de matière sèche contenue dans de la paille de mauvaise qualité de céréale d'hiver et $\mathrm{Io} \mathrm{kg}$ de matière sèche contenus dans des betteraves. Il conclut : "Il y aurait donc lieu de tenir également compte du coefficient de digestibilité de la matière sèche. "

BRÜGGEMAN (4) fait aussi quelques remarques (page 363) sur la matière sèche non digestible. A son avis, on ne peut faire une grosse erreur quand on laisse de côté la matière sèche non organique, qui ne forme qu'une partie relativement peu importante de la matière sèche. Dans les cas où la nourriture contient une grosse quantité de matières inorganiques, c'est qu'elle est sale et contient de la terre.

\section{D. - MES PROPRES RECHERCHES SUR LA QUESTION DU RASSASIEMENT DE LA VACHE A LAIT}

\section{I. - Réalisation technique de ces recherches :}

Les aliments étaient donnés aux vaches par petites quantités à la fois $2 \mathrm{~kg}$ d'aliment grossier volumineux ou $20 \mathrm{~kg}$ d'aliments aqueux succulent on évitait de donner à la fois des quantités trop fortes pour que l'aliment ne risque pas d'être chauffé et humidifié par la respiration de l'animal, ce qui aurait pu nuire au bon goût de l'aliment. C'est seulement quand un apport d'aliment avait été mangé qu'on faisait un nouvel apport. En principe les bêtes, comme cela a lieu généralement dans la pratique, étaient nourries deux fois par jour. La première alimentation avait lieu le matin à 7 heures et la deuxième l'aprèsmidi à $\mathrm{I} 5$ heures; la durée de chaque repas était de $\mathrm{I}$ à $\mathrm{I}, 5 \mathrm{~h}$. Cette durée variait suivant qu'il s'agissait d'un aliment grossier volumineux ou d'un aliment 
aqueux succulent. On observait attentivement les animaux, car seule leur attitude pouvait indiquer s'ils étaient rassasiés ou non. Quand on donne un aliment volumineux grossier (fourrage, paille) la vache est rassasiée assez rapidement. Quand on donne des aliments succulents, il s'écoule un temps plus long avant que la vache soit rassasiée. Il ne faut pas non plus se laisser tromper par le fait que la vache s'arrête de manger ; après un certain temps, assez court généralement, l'animal se met de nouveau à manger. La vache se comporte comme l'homme qui, après avoir absorbé une certaine quantité d'aliments a le sentiment d'être rassasié, puis après un temps de repos assez court ressent à nouveau une sensation de faim. L'alimentation des vaches était donc menée de manière à ce que les vaches soient rassasiées d'une manière durable et permanente. Aussitôt qu'une vache se couchait, commençait à ruminer et donnait a nsi, par son attitude générale une impression de satisfaction et de rassasiement, on ne lui donnait plus d'aliment. La partie laissée de l'aliment, les petits déchets de fourrage, les parties terreuses, les petites racines de betteraves étaient soigneusement rassemblés et pesés, et ce poids était déduit du poids de l'aliment donné...

Il ne suffisait pas de peser seulement l'aliment donné, il fallait encore déterminer exactement les substances nutritives absorbées. Pour cela, il était nécessaire de mesurer la digestibilité des aliments donnés. Ceci fut réalisé avec des moutons, à savoir deux moutons pour chaque aliment. Ces moutons étaient placés dans les stalles spéciales d'essais et recevaient des aliments identiques à ceux donnés aux vaches à lait. Pour les fourrages verts et l'ensilage, les essais avec les moutons étaient faits rigoureusement au même moment que ces aliments étaient donnés aux vaches, car ces aliments changent rapidement d'un jour à l'autre par suite de leurs variations rapides de composition et de teneur en eau quand la croissance progresse. Comme tous ces essais furent réalisés à l'automne, il est du reste probable que ces changements dûs à la croissance étaient relativement faibles, vu la lenteur de la croissance à cette saison. Par contre, les essais d'utilisation avec les moutons ne furent entrepris pour les aliments bruts (fourrage et paille) qu'après la fin des essais avec les vaches. Pendant tout le temps qu'on donnait ces aliments bruts aux vaches on prenait chaque jour un échantillon. Ces échantillons étaient hachés et donnés alors aux moutons, la quantité demeurant chaque jour la même à celle pesée au début de l'essai. La fiente des moutons était recueillie suivant la méthode connue du sac à fiente. Chaque essai d'utilisation avec les moutons durait 20 jours, dont ro jours étaient considérés comme une période préliminaire de préparation, et Io jours correspondant à l'essai réel. Les animaux étaient pesés pendant 3 jours consécutifs au début de la période préliminaire puis au début et à la fin de la période principale. Les poids indiqués à chaque essai d'utilisation (avec les moutons) sont les poids moyens au début de la période principale.

D'après les recherches faites précédemment à l'Institut de Kiel, qui du reste n'avaient rien à voir avec la question du rassasiement, il ressort que la 
vache à lait, lors d'un changement d'alimentation, dans le cas où le nouvel aliment ne lui est pas inconnu, se règle après quelques jours de manière à absorber une quantité déterminée de cet aliment ; et par la suite, mange avec régularité cette quantité qui demeure constante. D'après les essais de MOORE et Winter (39), la masse principale des éléments non digestibles de 1'aliment sort du système digestif après 22 à 33 heures. Il en résulte qu'une action ultérieure de la ration précédemment donnée sur le rassasiement ne peut guère se faire sentir après 2 jours. Aussi, une période de transition d'au moins 2 jours parut suffisante, en admettant bien entendu que les vaches s'étaient habituées à la nouvelle alimentation, ce qui du reste se traduisait par l'absorption quotidienne d'une quantité égale du nouvel aliment. Après ce temps de transition relativement court, la durée de l'essai lui-même ne pouvait pas être trop longue, puisque ces essais avaient seulement pour but de déterminer la quantité d'aliment qui rassasiait la vache et non de voir l'infuence de ces aliments sur la production de lait ou la variation de poids vif.

La plupart des essais furent faits avec des vaches pleines. En tout, il fut entrepris 5 séries d'essais avec 3 ou 4 vaches par série. I.es deux premières séries servirent comme essais préliminaires pour se rendre compte de la possibilité technique de réaliser ces essais. Puis quand ces deux premières séries eurent montré que notre technique était convenable nous poursuivimes les séries d'essais suivants.

\section{II. - Etude de chacun des essais : *}

\section{TABLEAU I}

Données sur les vaches ayant suivi mon essai:

(Les caractéristiques sont celles au début de la période des essais)

\begin{tabular}{|c|c|c|c|c|c|c|c|}
\hline & années & & mois & depuis & $\begin{array}{l}\text { Poids } \\
\mathrm{kg}\end{array}$ & $\begin{array}{l}\text { Produc- } \\
\text { tion de } \\
\text { lait en } \\
\quad \mathrm{kg}\end{array}$ & $\begin{array}{c}N^{o} \text { des essais } \\
\text { où elles ont été } \\
\text { utiiisées }\end{array}$ \\
\hline Gertrud . . . . . . . & 5 & & 8 & I 2 & 704 & seche & I, 2,3 \\
\hline 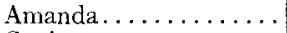 & 12 & & 8 & 2 & 685 & 1 & I; $\quad 2, \quad 3$ \\
\hline Genise . . . . . . . . . & 5 & & 8 & - & 640 & $n$ & I, $2, \quad 3$, \\
\hline Antonine ........... & 12 & & pas & leine & $5^{8} 5$ & $"$ & 4 \\
\hline Anna $\ldots \ldots \ldots \ldots$ & I 2 & & pas & leine & 569 & $n$ & \\
\hline Mulda $\ldots . . . \ldots \ldots$ & 4 & I I & 7 & 5 & 534 & I 2,0 & $5, \quad 6, \quad 7,8,9$ \\
\hline 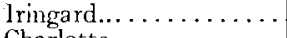 & 3 & J I & 4 & 7 & $5^{8} 3$ & I 2,0 & $5,6,7,8,9$ \\
\hline Charlotte ........... & 9 & I0 & 2 & 4 & 556 & I 3,0 & $5,6, \quad 7,8,9$ \\
\hline Jamaillsa ............ & 3 & 6 & 2 & .7 & 506 & $\mathrm{~T} 2,0$ & Iо, I I, I2, I3, I4, I 5 \\
\hline Ingrid $\ldots \ldots \ldots \ldots \ldots$ & 3 & 8 & 5 & -8 & 545 & 8,0 & Iо, I I, I 2, I 3, I 4, I 5 \\
\hline Irene $\ldots \ldots \ldots \ldots \ldots$ & 3 & 8 & 3 & 19 & 543 & $\mathrm{I} 4,0$ & $\mathrm{I}_{10}, \mathrm{I}, \mathrm{I} 2, \mathrm{I} 3, \mathrm{I}_{4}, \mathrm{I}_{5}$ \\
\hline Annemdrie $\ldots \ldots \ldots$. & II & 8 & 4 & 7 & 600 & I 3,0 & I0, I I, I $2, \mathrm{I} 3, \mathrm{I} 4, \mathrm{I} 5$ \\
\hline Heister............ & 4 & 8 & 3 & I I & 539 & I 4,0 & $16,17,18,19,20,2 \mathrm{I}$ \\
\hline Grasmüche $\ldots \ldots \ldots$. & 5 & 9 & 2 & 20 & 520 & $I_{5}, 0$ & 16, I7, 18, 19, 20, 2 I \\
\hline Gunda.............. & 5 & 7 & 4 & 24 & 564 & I 2,0 & $16,17,18,19,20,2 \mathrm{I}$ \\
\hline Gundel.............. & 6 & 7 & 5 & 19 & 510 & $I, 0$ & $16,17,18,19,20,2 \mathrm{I}$ \\
\hline
\end{tabular}

* Note du traducteur : J'ai laissé de côté toute cette vaste partie et donné seulement les tableaux I, II et III établis par moi. 


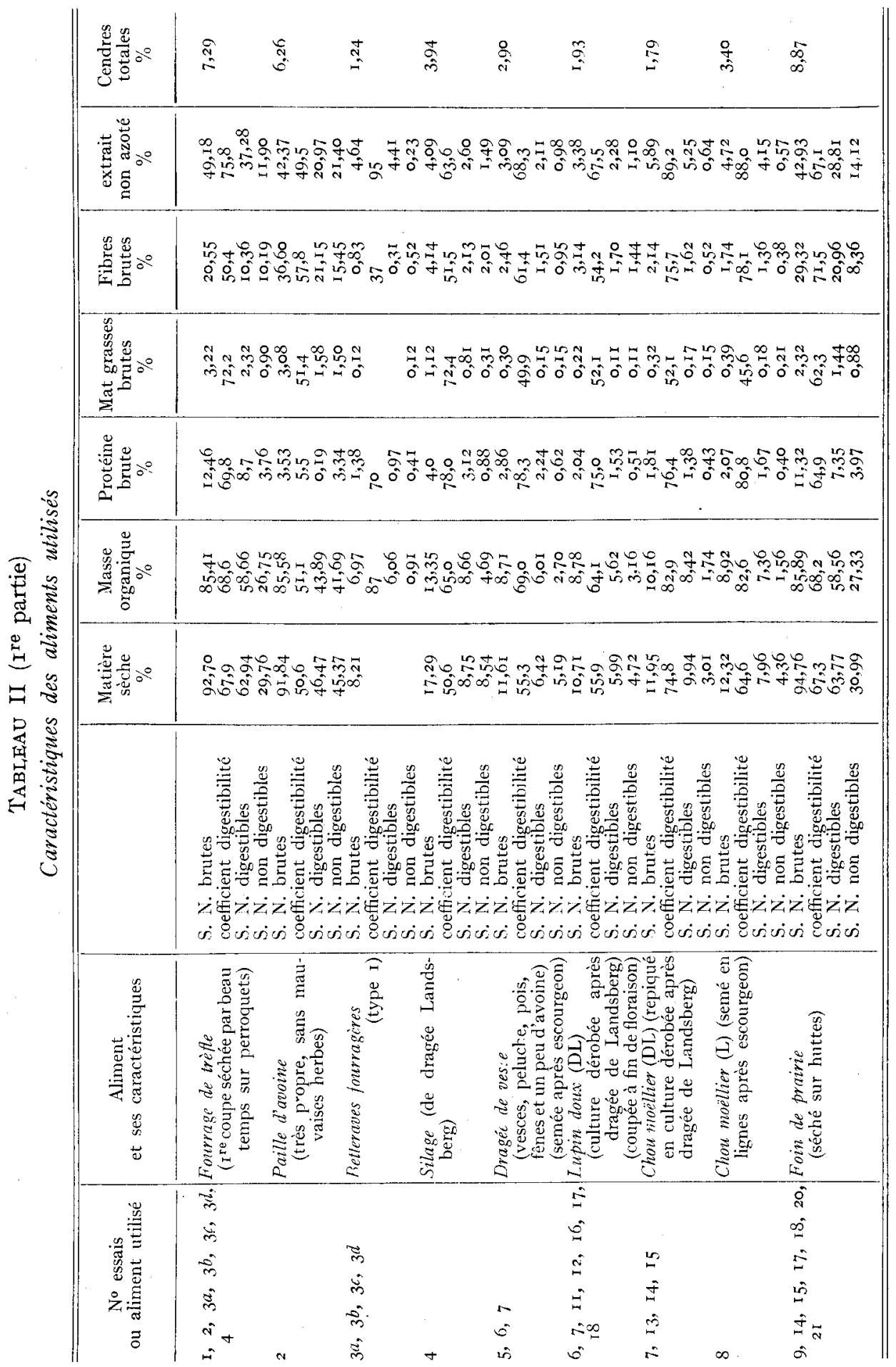


TABleAu II $\left(2^{\mathrm{e}}\right.$ partie $)$

Caractéristiques des aliments utilisés

\begin{tabular}{|c|c|c|c|c|c|c|c|c|c|}
\hline $\begin{array}{c}\text { No essai } \\
\text { ou ali- } \\
\text { ment } \\
\text { utilisé }\end{array}$ & $\begin{array}{c}\text { Aliment } \\
\text { et ses } \\
\text { caractéristiques }\end{array}$ & & 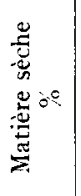 & 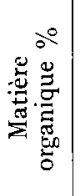 & 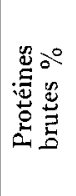 & 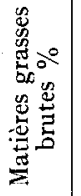 & 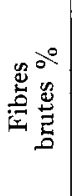 & 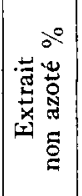 & 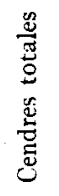 \\
\hline$\underset{22}{9, \text { II }}$ & $\begin{array}{l}\text { Betteraves fourra- } \\
\text { gères (type 2) }\end{array}$ & $\begin{array}{l}\text { S. N. brutes } \\
\text { coefficient digestibilité } \\
\text { S. N. digestibles } \\
\text { S. N. non digestibles }\end{array}$ & II, 28 & \begin{tabular}{r|}
10,40 \\
88 \\
9,20 \\
1,20
\end{tabular} & $\begin{array}{c}0,64 \\
70 \\
0,45 \\
0,19\end{array}$ & $\begin{array}{l}0,05 \\
0,05\end{array}$ & $\begin{array}{c}0,83 \\
37 \\
0,31 \\
0,52\end{array}$ & $\mid \begin{array}{c}8,88 \\
95 \\
8,44 \\
0,44\end{array}$ & 0,88 \\
\hline Io & $\underset{\text { Lupin doux }}{\text { (semé) }}$ (E) & $\begin{array}{l}\text { S. N. brutes } \\
\text { coefficient digestibilité } \\
\text { S. N. digestibles } \\
\text { S. N. non digestibles }\end{array}$ & $\begin{array}{c}8,09 \\
72,0 \\
5,82 \\
2,27\end{array}$ & $\begin{array}{c}6,85 \\
79,4 \\
5,43 \\
1,42\end{array}$ & $\begin{array}{c}\mathrm{I}, 78 \\
79,8 \\
\mathrm{I}, 42 \\
0,36\end{array}$ & $\begin{array}{c}0,20 \\
52,8 \\
0,10 \\
0,10\end{array}$ & $\begin{array}{r}1,80 \\
75,7 \\
1,36 \\
0,44\end{array}$ & $\mid \begin{array}{c}3,07 \\
83,2 \\
2,55 \\
0,52\end{array}$ & 1,24 \\
\hline $\begin{array}{l}9,19, \\
20,21\end{array}$ & $\begin{array}{c}\text { Chou moéllier (E) } \\
\text { (repiqué) }\end{array}$ & $\begin{array}{l}\text { S. N. brutes } \\
\text { coefficient digestibilité } \\
\text { S. N. digestibles } \\
\text { S. N. non digestibles } \\
\text { N. B. - S. N. = Subs }\end{array}$ & $\begin{array}{c}\mathrm{I} 1,64 \\
77,8 \\
9,06 \\
2,58\end{array}$ & $\begin{array}{c}9,57 \\
86,1 \\
8,24 \\
1,33\end{array}$ & $\begin{array}{c}2,36 \\
84,2 \\
1,99 \\
0,37\end{array}$ & $\begin{array}{c}0,37 \\
5 \mathrm{I}, 7 \\
0, \mathrm{I} \\
0,18\end{array}$ & $\begin{array}{r}\mathrm{I}, 73 \\
84,8 \\
\mathrm{I}, 47 \\
0,26\end{array}$ & $\left|\begin{array}{c}5, \mathrm{II} \\
89,9 \\
4,59 \\
0,52\end{array}\right|$ & 2,07 \\
\hline
\end{tabular}

Voir tableau III, page 84 .

\section{II. - Examen d'ensemble de tous les essais réalisés :}

Nous avons ainsi décrit et examiné rapidement chacun des essais faits pour observer les possibilités d'absorption des vaches nourries avec différents aliments. Les résultats de tous ces essais sont résumés dans le tableau III, page 84 .

Pour permettre une meilleure comparaison de ces essais, nous avons pris comme base 100 (de comparaison) les résultats de l'essai $n^{0} \mathrm{I}$ et y avons comparé les résultats des autres essais ramenés à Ioo $\mathrm{kg}$ de poids vif, tels qu'ils sont portés dans les colonnes IO-II-I2-I3. Cette comparaison par rapport à l'essai $n^{0} \mathrm{I}$ est indiquée dans les colonnes I4-I5-I6-I7.

On remarque immédiatement que la matière sèche et le ballast absorbés sont particulièrement bas dans les essais $2,8,13$, I4 et 19 . Il a déjà été noté dans la discussion des essais individuels que les aliments utilisés pour la ration de rassasiement n'étaient pas volontiers mangés par les vaches. Il s'agissait de paille d'avoine et de trois espèces de choux moëlliers (plantés après dragée Landsberg, semés en ligne après escourgeon, plantés après escourgeon). Les bêtes ne mangeaient de ces aliments que ce qu'il leur fallait pour satisfaire leur appétit. Il fut alors absorbé des quantités de matière sèche qui étaient de $5 \mathrm{I} \%$ de la consommation maxima obtenue dans l'essai $\mathrm{n}^{0} 5$. Les différences sont encore plus grandes en ce qui concerne le ballast absorbé. Celui-ci dans l'essai $\mathrm{n}^{\circ}$ I 9 correspond à $26 \%$ de l'absorption de base de l'essai $\mathrm{n}^{0}$ I. La différence 


\begin{tabular}{|c|c|c|c|}
\hline tessa il $ә \mathrm{p} \cap \mathrm{N}$ & & & 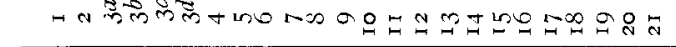 \\
\hline iseireg & $a^{2}$ & $\approx$ & 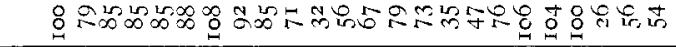 \\
\hline 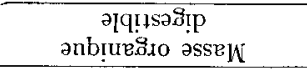 & $\therefore$ & $\stackrel{2}{2}$ & 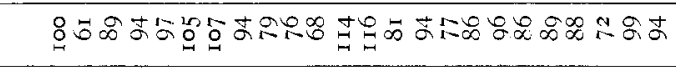 \\
\hline 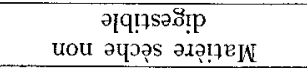 & $\partial^{2}$ & $\stackrel{i n}{\rightarrow}$ & 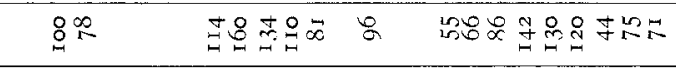 \\
\hline 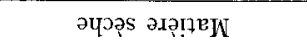 & $\circ$ & \pm & 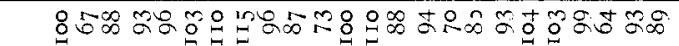 \\
\hline 䓀 & $\stackrel{00}{-10}$ & $=$ & 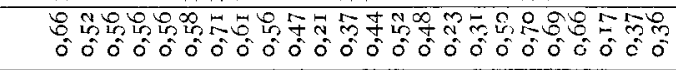 \\
\hline 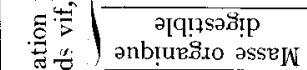 & 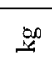 & $\stackrel{9}{-1}$ & 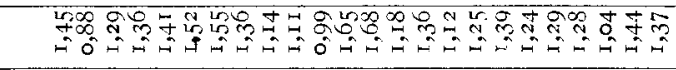 \\
\hline 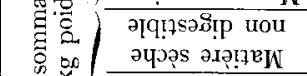 & 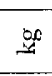 & $\Xi$ & 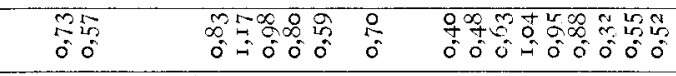 \\
\hline 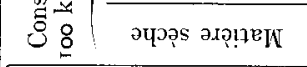 & 5 & 9 & 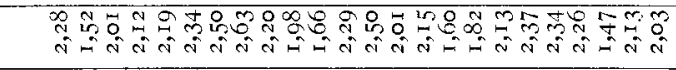 \\
\hline equosqe isel[eg & 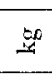 & 0 & 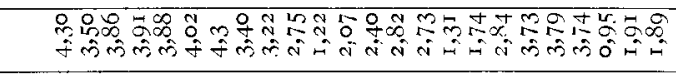 \\
\hline 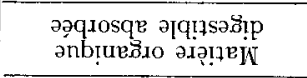 & 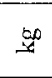 & $\infty$ & 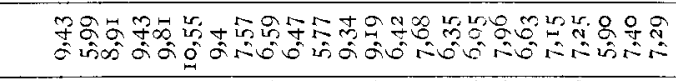 \\
\hline 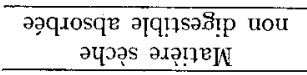 & $\stackrel{\infty 0}{-4}$ & r & 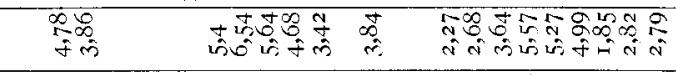 \\
\hline 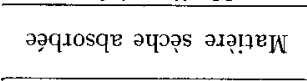 & $\approx$ & 6 & 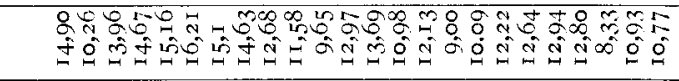 \\
\hline xneuịue səp spiod & $\underset{x}{x}$ & n & 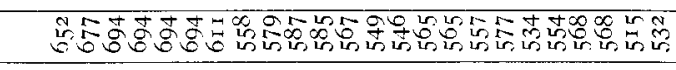 \\
\hline 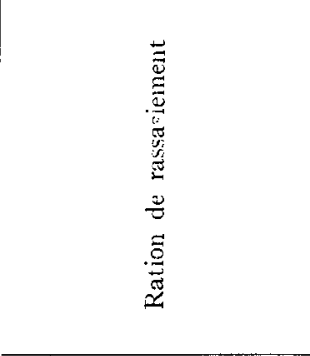 & $\underset{x}{a x}$ & + & 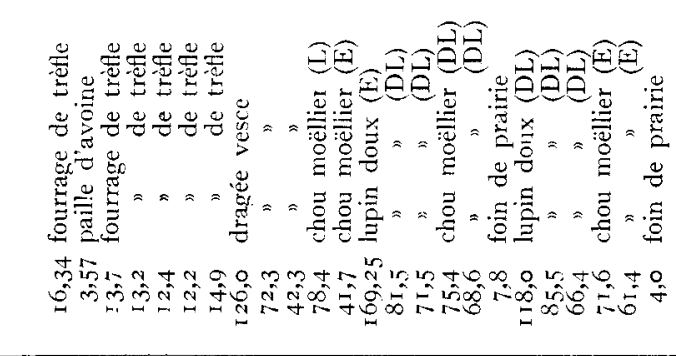 \\
\hline 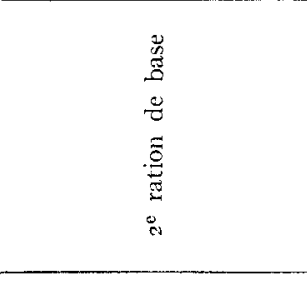 & $\underset{c}{x}$ & $m$ & 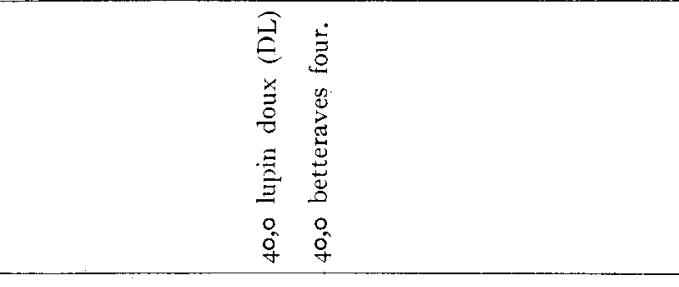 \\
\hline 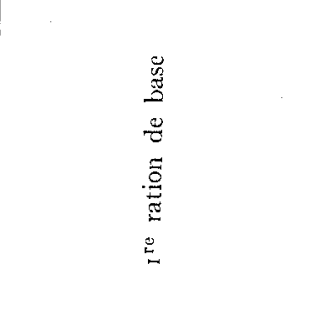 & 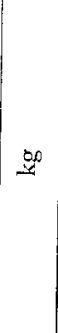 & $m$ & 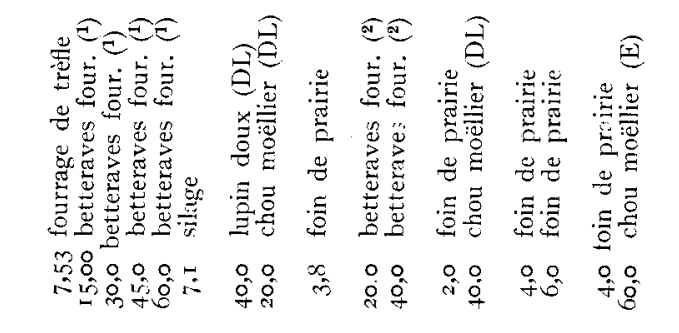 \\
\hline epssa i әp oN & & H & 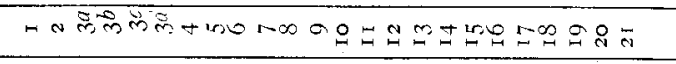 \\
\hline 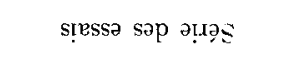 & & & - $\quad \exists \Xi \quad \geq \quad>$ \\
\hline
\end{tabular}


entre le ballast absorbé dans cet essai no i 9 et l'absorption maxima de ballast obtenu dans l'essai n ${ }^{\circ} 4$ (silage et foin de trèfle) est donc de $82 \%$

On peut admettre que, d'après la consommation d'aliments indiqués dans le tableau, les vaches n'étaient pas encore complètement rassasiées ; c'est ce qu'ont montré les essais complémentaires des essais I3 et I9. Par exemple, dans l'essai 13 où on donna pour atteindre le rassasiement $73,4 \mathrm{~kg}$ de choumoëllier ce qui correspondait à l'absorption d'une quantité relativement faible de matière sèche et de ballast, il $\mathrm{y}$ eut une augmentation notable de ces deux éléments absorbés quand on donna seulement $2 \mathrm{~kg}$ de foin de prairie (essai $n^{0}$ I4). I' 'absorption de matière sèche et ballast s'éleva encore quand on réduisit à $40 \mathrm{~kg}$ la consommation de chou-moëllier, aliment mangé peu volontiers par les vaches, et en l'utilisant comme première ration de base, le foin de prairie devenant la ration de rassasiement. C'est alors seulement dans l'essai $n^{\circ}$ I5 que les vaches ont alors probablement mangé jusqu'à la limite de leur capacité d'absorption. Alors que le faible bon goût d'un aliment avait pour résultat que l'absorption de matière sèche était très faible ; le fait de remplacer une partie de cet aliment peu apprécié par un autre à bon goût pour les vaches a pour résultat d'augmenter l'absorption de ballast et surtout de matière sèche. Il était du reste facile, en observant l'avidité avec laquelle les bêtes mangeaient leur ration et le temps qu'elles mettaient à la manger de voir si un aliment était mangé volontiers ou pas, par les vaches. Les aliments mangés volontiers étaient le fourrage de trèfle, les betteraves, la dragée de vesce et le lupin doux. Les choux moëlliers semés en ligne après escourgeon et qui, au moment où ils étaient donnés à manger, étaient encore dans un stade de végétation peu avancé étaient également bien mangés. La forte absorption de matière sèche et atu moins partiellement de ballast dans les essais I, 5, I0, I6, I7 et I 8 montrent nettement que les aliments donnés dans ces essais paraissaient d'un bon goùt aux vaches. Dans tous les essais, il fut absorbé plus de matière sèche que dans l'essai de base $n^{\circ}$ I ; dans les essais I6, I7 et I 8 il fut également absorbé plus de ballast que dans 1'essai de base $n^{\circ}$ I. On peut donc déduire de ces essais sur le rassasiement des vaches la preuve du principe suivant : La quantité d'aliment consommée dépend avant tout du bon goût de ces aliments.

Il est intéressant d'examiner dans ces essais la quantité de masse organique digestible absorbée. Nous vovons que cette quantité dépend d'abord de la teneur de l'aliment en masse organique digestible mais aussi beaucoup du goût plus ou moins bon de l'aliment. La masse organique digestible contient les substances nutritives qui permettent l'entretien et la production. Dans le cas de l'alimentation avec des produits de la ferme, la question fondamentale est de savoir la limite de production que nous permettent d'obtenir ces aliments. La question peut également se poser de la manière suivante : quelles sont les quantités maxima d'aliments produits à la ferme que peuvent absorber nos vaches et quelles sont les productions que permettront les substances nutritives contenues dans la quantité d'aliments absorbée ? De nos recherches il 
ressort un fait dont il est indispensable de tenir compte si nous voulons obtenir la production maxima avec les aliments de la ferme : nous devons avant tout veiller à établir nos rations pour que leur goût plaise aux vaches et qu'elles aient ainsi tendance à en absorber le plus possible. Il est donc indispensable que nous sachions quels sont les aliments que nos vaches mangent avec plaisir et dont, par conséquent, nous serons en mesure de leur faire absorber de grosses quantités. Inversement, nous devons savoir quels sont les aliments qui ne sont pas mangés volontiers par les vaches et dont, par suite, elles n'absorberont jamais de grosses quantités. Les aliments dont le goût est peu apprécié de la vache doivent être donnés en quantités limitées comme ration de base, et les aliments au bon goût seront donnés comme ration de rassasiement. Reprenons les essais I3, I4 et I5. Le remplacement partiel d'une partie du chou moëllier employé comme ration de rassasiement par du foin de prairie en ration de base a augmenté l'absorption de masse organique digestible de $\mathrm{I}, \mathrm{I} 2 \mathrm{~kg}$ à $\mathrm{I}, 25 \mathrm{~kg}$ (par Ioo $\mathrm{kg}$ de poids vif) (essais $\mathrm{I}_{3}$ et $\mathrm{I} 4$ ). Mais quand, dans l'essai no $\mathrm{n}_{5}$, le chou moëllier devient ration de base et le foin de prairie ration de rassasiement, l'absorption de matière organique digestible s'élève à $\mathrm{I}, 39 \mathrm{~kg}$, ce qui représente une augmentation de $24 \%$ par rapport à l'essaj $n^{0}$ I3 et de II \% par rapport à l'essai no $\mathrm{I}_{4}$, De même, dans d'autres essais ( $\mathrm{n}^{\circ} 3$, I I et I2) une augmentation de la quantité donnée de betteraves a élevé la quantité de masse organique digestible absorbée. Les betteraves ainsi qu'un ensilage de bonne qualité (voir essai $n^{\circ} 4$ ) sont toujours mangés volontiers par les vaches. On peut faire absorber aux vaches $60 \mathrm{~kg}$ de betteraves et, en plus, une bonne ration de foin (essai $\left.\mathrm{n}^{\circ} 3 d\right)$.

Hansson (23) a montré l'influence de la qualité des fourrages sur la quantité absorbée. Pendant 4 années consécutives les mêmes vaches reçurent en plus de $30 \mathrm{~kg}$ de, betteraves fourragères et $\mathrm{I}, 2 \mathrm{~kg}$ d'avoine moulue la quantité de fourrage voulue pour les rassasier. Hansson put alors constater que la quantité moyenne de fourrage consommé s'éleva de $12,0 \mathrm{~kg}$ en $1932-33$ à $13,5 \mathrm{~kg}$ en r935-36. On considéra que la cause de cette augmentation était l'amélioration de qualité due à ce qu'on coupait la plante quand elle était plus jeune. Il en résulte une double action importante résultant de cette amélioration de qualité : d'abord, pour le même poids de fourrage, les bêtes absorbent une quantité plus élevée de substances nutritives; ensuite, elles mangent une quantité plus élevée de ce fourrage auquel elles trouvent un meilleur gô̂t, ce qui cause une deuxième augmentation des substances nutritives absorbées. Si on veut obtenir l'absorption maxima de substances nutritives avec les aliments de la ferme, il est important de se rappeler que la quantité d'aliments absorbés dépend beaucoup du bon goût de ces aliments.

Il s'agit maintenant d'essayer de savoir quels sont les constituants des aliments qui donnent à la vache le sentiment d'être rassasiée. Nous estimons que nous devons éliminer de nos considérations les essais dans lesquels on utilisa des aliments que les vaches ne mangeaient pas volontiers. En effet, dans ces 
essais, la quantité consommée d'aliments était réduite, non pas à cause de certains constituants de l'aliment, mais parce que leur goût répugnait plus ou moins à la vache.

Nous éliminerons donc les essais 2,8, I3, I4 et I9. Nous voyons alors dans les autres essais, comme le montre la colonne $I_{4}$, que l'absorption de matière sèche ne subit pas de variations importantes aussi bien dans les séries d'essais avec les mêmes vaches qu'avec des vaches différentes au cours des diverses séries d'essais. On peut observer qu'avec certains aliments l'absorption de matière sèche est élevée. C'est le cas dans l'essai $n^{0} 5$ avec une dragée de vesce et dans l'essai $\mathrm{n}^{0}$ ro avec le lupin doux; malgré tout, les variations de matière sèche absorbée ne dépassent pas I5 \% en plus ou en moins du chiffre de base Ioo dans l'essai $\mathrm{n}^{\circ} \mathrm{I}$.

Cependant l'absorption de matière sèche comme on peut le voir d'après les chiffres des essais faits avec les mêmes vaches recevant des aliments différents n'est pas une quantité constante, mais varie dans certaines limites, qui dépendent avant tout du goût de l'aliment.

Il faut souligner qu'une forte teneur en eau dans un aliment n'empêche pas l'absorption d'une importante quantité de matière sèche. Nous revenons, à ce sujet, aux remarques que nous avons faites au sujet des essais $3 a, b, c$ et $d$ et aux essais Io, II, et I2. Dans l'essai $3 a$, l'absorption de matière sèche diminua d'abord, relativement à l'essai de base $\mathrm{n}^{0} \mathrm{I}$, du fait qu'une partie de l'alimentation unique en trèfle avait été remplacée pour une petite partie par des betteraves. Mais quand on quadrupla cette quantité de betteraves (essai $n^{0} 3 d$ ) on n'observa pas une diminution de la matière sèche absorbée, mais au contraire une augmentation. On put faire la même observation dans les essais II et 12 ; un faible apport de betteraves amena d'abord une baisse de la quantité de matière sèche absorbée ; un apport plus fort augmenta à nouveau cette quantité. Dans l'essai no Io, les vaches absorbèrent $169 \mathrm{~kg}$ de nourriture verte contenant $155 \mathrm{~kg}$. d'eau. Si l'eau de la plante avait une action rassasiante, les vaches n'auraient certainement pas été en mesure d'absorber $\mathrm{I}_{4} \mathrm{~kg}$ de matière sèche. La quantité de matière sèche absorbée dans cet essai et l'essai no 5 (avec dragée de vesce) sont les quantités les plus élevées absorbées par Ioo $\mathrm{kg}$. de poids vif que nous ayons observées au cours de tous nos essais. Il n'existe aucune différence sensible entre les quantités de matière sèche absorbée au cours des essais, où on utilisait un fourrage sec mangé avec plaisir par les vaches (fourrage de trèfle) et contenant $7,3 \%$ d'eau, ainsi qu'au cours des essais faits avec des fourrages également mangés avec avidité et contenant $92 \%$ d'eau. Ces essais nous permettent donc de faite les affirmations suivantes :

I Sous réserve $\mathrm{du}$ bon goût de l'aliment, la quantité de matière sèche absorbée demeure un bon élément de mesure de la capacité d'absorption de la vache.

$2^{\circ}$ L'eau contenue dans les aliments ne diminue pas la quantité de matière sèche absorbée, laquelle demeure la même avec des fourrages secs ou des 
aliments aqueux, à condition que les deux espèces d'aliments soient également mangées avec plaisir par les vaches.

Au cours de nos essais, la quantité de matière sèche absorbée par des vaches de 550 à $700 \mathrm{~kg}$ varia entre 8 et $16 \mathrm{~kg}$. La quantité la plus faible fut observée dans le cas d'aliments mangés sans plaisir par les vaches. Les bêtes d'un poids de $55^{\circ}-600 \mathrm{~kg}$ absorbèrent I I à $\mathrm{I} 4 \mathrm{~kg}$ de matière sèche et celles d'un poids de 600-700 kg absorbèrent jusqu'à $16 \mathrm{~kg}$ de matière sèche. Les chiffres observés par nous correspondent donc à peu près à ceux que l'on trouve dans la littérature, et qui, comme nous l'avons dit, varient dans d'assez fortes limites.

La quantité absorbée de matière sèche non digestible ne put être déterminée dans le cas des essais faits avec les betteraves, car on ne put faire des essais d'utilisation réelle avec les betteraves. Les chiffres concernant la digestibilité de la matière sèche globale (organique et non organique) manquent dans la littérature pour tous les aliments. A dire vrai, il faut avouer, qu'il est très difficile de donner des chiffres précis pour la digestibilité de la matière sèche globale. Quand on essaie de déterminer la digestibilité de la matière sèche on est obligé de considérer en même temps les éléments non organiques de l'aliment étudié aussi bien que les éléments organiques. Dans le cas des dragées de vesce ou du lupin doux il était impossible d'éviter que le fourrage ne vint au contact de la terre ou du sable, et quoique ceci fût à peine observable, la plante verte était donnée plus ou moins souillée à l'animal. L'analyse révéla du reste une teneur en sable de I2 \% dans la dragée de vesce et de $8 \%$ dans le lupin doux. Il en résulte que dans les essais $5,6,7, \mathbf{I} 6, \mathrm{I} 7$ et $\mathrm{I} 8,1$ 'absorption de matière sèche non digestible est anormalement très élevée. Les chiffres des autres essais montrent d'ailleurs des variations considérables, de sorte que l'on est en droit de douter que ce chiffre puisse fournir un critérium permettant de juger du rassasiement de l'animal. Même dans les essais d'une même série (essais 16 , I7, I8 de la série $V$ ) où la quantité de matière sèche absorbée resta remarquablement constante, la quantité de matière sèche globale non digestible absorbée varia considérablement et on observa des différences allant jusqu'à $22 \%$. Nous déduirons donc de ces essais que la quantité de matière sèche globale non digestible absorbée ne permet pas de juger l'action rassasiante d'un aliment.

La quantité de ballast absorbé put être très exactement déterminée dans chacun des essais, car la masse organique non digestible de tous les aliments pouvait être exactement déterminée par les essais d'utilisation réelle. Comme nous 1'avons déjà dit, les betteraves seules ne furent pas soumises à ces essais d'utilisation réelle, car cela nous parut inutile, vu la très forte digestibilité des substances nutritives qu'elles contiennent. La teneur en ballast des betteraves, si on applique les coefficients de digestibilité indiqués par KELINER, sont de $\mathrm{I}, 28$ ou $\mathrm{I}, 20 \%$.

Les quantités absolues ou relatives de ballast absorbé qui furent observées 
au cours de nos essais, sont indiquées dans les colonnes 9, I3 et I7 du tableau III. Nous devons également, dans ce cas, laisser de côté les essais où on utilisa des aliments qui n'étaient pas volontiers mangés par les vaches, c'est-à-dire les essais 2, 8, I3, I4 et I9.

Dans la série d'essais I, la différence de ballast absorbé par les mêmes vaches dans le cas où elles recevaient seulement du fourrage de trèfle et dans le cas où elles recevaient des betteraves et du trèfle est de $\mathbf{1}_{5} \%$. Dans la série d'essais III la différence dans le cas de vaches identiques est de $36 \%$ (essai 5 comparé avec l'essai 9).

Dans la série d'essais IV (où on élimine les essais I3 et I4 comme nous l'avons dit) les différences de ballast absorbé sont de I2\% (essai ro comparé à l'essai II). Contrairement aux essais I, $3 a, 3^{b}, 3^{c}$ et $3^{d}$ (de la série I) l'introduction de betteraves dans l'essai i I amena par rapport à l'essai Io une augmentation de la quantité de ballast absorbé. alors que dans les essais cités de la série I la quantité de ballast absorbé avait diminué de I5\% par suite de l'introduction de betteraves dans la ration. L'apport de betteraves dans l'essai $3 d$ avait très légèrement relevé (de $3 \%$ ) l'absorption de ballast; or, dans l'essai I2, quand on continue à augmeutter la quantité de betteraves données, le ballast absorbé, qui avait d'abord cru, diminue de $6 \%$.

Dans ces mêmes essais, la quantité de matière sèche organique absorbée reste au contraire beaucoup plus stable, quoique l'introduction d'une faible quantité de betteraves dans la ration diminua d'abord légèrement la quantité de matière sèche absorbée ; puis, l'introduction d'une plus grande quantité de betteraves augmenta à nouveau la quantité de matière sèche absorbée.

Dans la série d'essais V (où on élimine l'essai I9, comme déjà dit) les différences de ballast absorbé sont également importantes. Les mêmes vaches qui, dans l'essai I6 avec lupin doux seul avaient absorbé $0,70 \mathrm{~kg}$ de ballast par Ioo $\mathrm{kg}$ de poids vif absorbèrent dans l'essai 2 I seulement $0,36 \mathrm{~kg}$ de ballast. Ceci représente une différence de $52 \%$ entre les essais I6 et $2 \mathrm{I}$. Par contre, on observe, une bonne régularité du ballast absorbé dans les essais I6, I7 et I 8 ; mais l'examen de la colonne $\mathrm{I} 4$ nous montre que la quantité de matière sèche absorbée ne subit pas de grosses variations; de plus, dans les essais 20 et $2 \mathrm{I}$, si la quantité de matière sèche diminue très sensiblement, elle diminue malgré tout relativement beaucoup moins que le ballast absorbé. En effet, dans l'essai 2 I relativement à l'essai $\mathbf{6} 6$, la matière sèche absorbée a diminué de I $_{5} \%$, alors que le ballast absorbé avait diminué de $52 \%$.

Le fait que, dans les colonnes I4, I5, I6 et I7 les résultats des essais sont calculés proportionnellement aux résultats de l'essai I pris comme base roo a facilité les comparaisons. La colonne I7, comme nous l'avons déjà dit, montre clairement les variations importantes du ballast consommé par roo $\mathrm{kg}$ de poids vif. Alors que la variation de matière sèche organique absorbée varie d'environ I5\% (si on élimine, comme déjà dit, les essais 2,8 , I3, I4 et I9) le ballast absorbé subit des variations allant jusqu'à $46 \%$ (essai 2 I comparé 
à l'essai I). C'est seulement dans l'essai i 8 qu'on observe une absorption de ballast égale à celle de l'essai de base $\mathrm{n}^{\circ} \mathrm{I}$. Dans trois cas (essais $4, \mathrm{I} 6$ et I7) le ballast absorbé est supérieur à celui absorbé dans l'essai de base $n^{\circ} \mathrm{I}$. Dans tous les autres cas, la quantité de ballast absorbé est nettement inférieure à celle de l'essai de base $n^{\circ} \mathrm{I}$, essai dans lequel la quantité absolue de ballast absorbé fut rigoureusement de $4,3 \mathrm{~kg}$ norme indiquée par LEHManN. Il est $\mathrm{du}$ reste bon de noter que, dans cet essai $\mathrm{n}^{0} \mathrm{I}$, le poids moyen des bêtes était de $652 \mathrm{~kg}$ alors que LeHMANN indique un chiffre de ballast absorbé de 4,3 $\mathrm{kg}$ pour des bêtes d'un poids moyen de $500 \mathrm{~kg}$.

Je crois donc pouvoir déduire de mes essais les conclusions suivantes :

$I^{\circ}$ La quantité de ballast absorbé fut de $4,3 \mathrm{~kg}$ dans deux essais seulement sur $2 \mathrm{I}$ essais. Ce chiffre standard fut atteint (essais I et 4 ) avec des bêtes d'environ $600 \mathrm{~kg}$ de poids vif; il semble donc que le chiffre de $4,3 \mathrm{~kg}$ de ballast absorbé pour des bêtes de $500 \mathrm{~kg}$ de poids vif est trop élevé. Dans tous les cas, ce chiffre du ballast absorbé a des valeurs très différentes, comme l'ont montré nos essais.

$2^{0}$ Avec des vaches identiques, la quantité de ballast absorbé est loin d'être constante, et au contraire varie considérablement suivant les aliments fournis.

La quantité de ballast absorbé est influencée par:

a) Le bon goût du fourrage.

Quand on fournit à la vache des aliments qu'elle ne mange pas volontiers, la quantité d'aliment absorbé et en même temps la quantité de ballast absorbé diminuent énornément.

Exemple: essai $\mathrm{n}^{\circ} 2$ avec paille d'avoine, essais no 8 , I3 et 19 avec chou moëllier. Au contraire, si on fournit en même temps à la vache des aliments qu'elle mange plus volontiers, la quantité de ballast absorbé augmente.

Exemples : comparaison des essais $\mathrm{n}^{0} 7$ et 8 , comparaison des essais $\mathrm{n}^{0}$ I4 et I5).

b) La teneur en ballast des aliments :

Quand on donne à la vache des aliments riches en ballast la quantité de ballast absorbé est plus élevée qu'avec des aliments pauvres en ballast.

Exemple: essai $n^{0}$ I (fourrage de trèfle) comparé aux essais $n^{0} 3$ (betteraves) $\mathrm{n}^{\mathrm{o}}$ Io où on donne uniquement du lupin doux, plante ayant la très faible teneur en ballast de $I, 42 \%$, alors que dans l'essai $n^{0}$ I6 on fournit uniquement du lupin doux, mais qui a une teneur en ballast beaucoup plus élevée, à savoir 3,I6\%, ce qui amène par Ioo $\mathrm{kg}$ de poids vif une absorption de $0,44 \mathrm{~kg}$ de ballast dans l'essai $\mathrm{n}^{0}$ Io, contre $0,70 \mathrm{~kg}$ dans l'essai $\mathrm{n}^{0} \mathrm{x} 6$. Dans l'essai $n^{0} 5$, on emploie uniquement une dragée de vesce dont la teneur en ballast est de $2,70 \%$ c'est-à-dire est située entre les teneurs du lupin doux des essais ro et I6 ; nous trouvons alors par Ioo $\mathrm{kg}$ de poids vif une absorption de ballast de $0,6 \mathrm{I} \mathrm{kg}$ qui se place également entre les chiffres des essais io et 16 . 
En ce qui concerne l'influence des substances organiques non digestibles c'est-à-dire du ballast, sur l'effet rassasiant des aliments de la vache, nos essais nous permettent de dire qu'il ne nous paraît pas possible d'utiliser la notion de ballast comme élément pour juger la quantité d'aliment absorbée par la vache. C'est seulement dans quelques cas isolés que nous avons observé l'absorption de la quantité standard de $4,3 \mathrm{~kg}$ de ballast. Nous n'avons trouvé aucune confirmation de l'hypothèse qu'une vache absorbe toujours la même quantité de ballast et que ce chiffre de la quantité de ballast absorbé est constant pour la même vache. Il ne nous paraît donc pas possible de considérer comme valable, tout au moins d'une manière aussi absolue, la conception jusqu'ici admise dans les théories sur l'alimentation que le ballast permet de mesurer la valeur rassasiante d'un aliment et les besoins d'une vache pour être rassasiée. La teneur en ballast d'un aliment nous paraît donc seulement intéressante en ce que la présence d'une certaine quantité de ballast dans la ration des ruminants paraît absolument nécessaire pour permettre un bon fonctionnement des organes digestifs. La teneur en ballast a également l'avantage de bien faire apparaître la valeur nutritive plus ou moins grande d'un aliment, ainsi que sa digestibilité.

Nos essais nous amènent à conclure que c'est avant tout la teneur en matière sèche des aliments qui détermine chez la vache le sentiment d'être rassasiée. Ceci est prouvé par le fait que les vaches après l'absorption d'une quantité de matière sèche correspondant à celle observée dans l'essai de base $n^{0} I$, arrêtèrent de manger, quoiqu'à ce moment, la quantité de ballast absorbé était très inférieure à celle observée dans l'essai de base $n^{\circ}$ I. Au cours de plusieurs de ces essais, il est apparu nettement que l'action rassasiante ne pouvait provęnir que de la matière sèche absorbée, car la quantité de ballast absorbée était si faible qu'elle ne pouvait certainement pas causer une action rassasiante. Comme exemple nous citerons les essais $3 d, 6$, IO, I2, I5, 20 et $2 \mathrm{I}$, où la quantité de matière sèche absorbée était à peu près égale à celle absorbée dans l'essai de base $n^{\circ} \mathrm{r}$, mais où au contraire la quantité de ballast absorbée était nettement inférieure à celle de l'essai de base $n^{\circ}$ I. Quand les aliments donnés sont très différents en ce qui concerne la teneur en matière sèche, eau, ballast, matière sèche non digestible, c'est la quantité de matière sèche absorbée qui montra les variations les plus faibles. Il semble donc que c'est la matière sèche qui est l'élément le plus sûr pour juger de l'action rassasiante d'un aliment et, dans tous les cas, représente un élément beaucoup plus sûr que tous ceux jusqu'ici proposés et essayés. Le bon goût de l'aliment joue un rôle important et influence la quantité de matière sèche absorbée ; car la vache absorbera une quantité plus importante de matière sèche si le goût de l'aliment qui lui est présenté lui convient, alors que si elle mange peu volontiers un aliment, elle absorbera une quantité de matière sèche plus faible.

L'utilisation de la matière sèche présente également l'avantage par rapport au ballast que chacun se représente plus facilement la teneur en matière sèche 
d'un aliment et comprend plus facilement ce chiffre que celui du ballast. Les variations de teneur en matière sèche dans les aliments de même espèce, par exemple des fourrages de diverse qualité sont moins importantes que les variations de la teneur en ballast, ce qui rend beaucoup plus difficile de s'en servir comme base de rassasiement. Le bon goût de l'aliment jotie le même rôle sur la quantité de matière sèche absorbée que sur la quantité de ballast absorbée. Mais alors que la teneur en matière sèche des aliments influe assez peu sur la quantité de matière sèche absorbée, la teneur en ballast des aliments exerce une influence très nette sur la quantité de ballast absorbée, comme l'ont montré nos essais.

Les conclusions de nos essais se trouvent confirmées quand nous examinons les processus physiologiques qui se développent dans le corps de l'animal an cours de l'absorption, de la digestion, des aliments.

Au cours de l'absorption des aliments, les bouchées mélangées de salive arrivent dans le bonnet et la panse (MANGOLD 37). Or, d'après SCHEUNERT et Steinhouf la progression du contenu de la panse dans le système digestif dépend de :

$I^{0}$ de la teneur en eau;

$2^{0}$ du degré de division de l'aliment;

$3^{0}$ de la quantité contenue dans la panse.

D'après Scheuner't, la progression de la masse fluide dépend du degré de remplissage de la panse et de la teneur en fluide de la panse. La masse fluide absorbée sera retenue, si la panse est relativement vide et sèche. Au contraire la masse sera rapidement portée en avant si la panse est bien remplie avec un contenu suffisamment fluide. Il en résulte alors une digestion de l'estomac d'une régularité remarquable (ELLENBERG et SCHEUNERT I3). L'estomac forme en quelque sorte une "nourrice d'alimentation " et un "volant de nourriture " qui prépare la nourriture en la gonflant, l'amollissant et lui enlevant certains éléments extractifs. Il fournit ensuite à l'intestin, d'une manière continue, des petites portions de masse alimentaire, qui permettent à l'intestin d'être rempli comme il faut pour qu'il digère convenablement, tout en évitant que l'intestin soit trop rempli. C'est le degré de remplissage de l'estomac qui détermine si un être vivant se sent rassasié ou a faim. C'est dans l'estomac que se prépare la digestion, qui se réalise dans l'intestin. Dans le cas des ruminants, le temps principal de séjour de la nourriture dans le système digestible est dans l'estomac.

D'après Disselhorst (I2) les processus de fermentation et de désagrégation se continuent dans le gros intestin. C'est dans la partie terminale de l'intestin des végétivores que se fait la digestion de la cellulose et de l'hémicellulose, phénomène au cours duquel les bactéries jouent un rôle important. La cellulose est dissoute et transformée en acides gras et produits gazeux. Grâce à cette dissolution de la paroi des cellules des aliments végétaux (les setuls mangés par les vaches laitières) le précieux contenu de ces cellules, s'il 
n'a pas déjà été mis à nu par la désintégration mécanique due au mâchage et à la rumination, peut alors subir l'action des ferments digestifs.

Ainsi, dans la première partie des organes digestifs de la vache, il ne se produit pas une absorption totale de toutes les substances nutritives digestibles de la ration, mais une grande partie de ces substances, et probablement une partie qui est loin d'être faible, est seulement dans la partie terminale de l'intestin digérée et transmise au circuit sanguin.

Aussi longtemps que les parties digestibles se trouvent mélangées avec les parties non digestibles, organiques ou non organiques, dans le système digestif du ruminant, elles exercent une pression sur les parois des organes de ce système, autrement dit, elles ont une action rassasiante. En effet, comme LEHMANN 1'a observé, la tension des parois abdominales joue un rôle important dans la création du sentiment de rassasiement chez l'animal. Il n'est donc pas possible de dire que seules les parties non digestibles de l'animal créent chez la vache le sentiment d'être rassasiée.

La considération du mécanisme de la digestion chez les ruminants confirme donc les observations faites au cours de nos essais, à savoir que le sentiment de rassasiement est déterminé par la quantité de matière sèche absorbée. Cette matière sèche comprend les parties digestibles et non digestibles, organiques ou non organiques, de l'aliment. L'étude de la question du rassasiement, vue du point de vue physiologique, confirme la conception, qui se dégage de nos essais, à savoir que c'est la quantité de matière sèche absorbée qui détermine pour la vache le sentiment de rassasiement.

\section{E. - RESUMÉ}

Pour nourrir les vaches à lait, nous devons aujourd'hui utiliser le plus possible les aliments produits à la ferme. Il est donc important de déterminer la limite de production que nous permettent ces aliments. Une réponse ne peut être donnée que si nous déterminons les quantités maxima de ces aliments que les vaches peuvent absorber. Nous devons donc essayer de trouver un élément de base qui nous permette de mesurer l'action rassasiante des aliments et les besoins de la vache pour être rassasiée. Le but d'une alimentation rationnelle est donc de donner à la vache une alimentation quii la rassasie, sans surcharger son organisme, mais tout en lui fournissant les substances nutritives dont elle a besoin.

Nous voulions, en particulier savoir si on peut utiliser comme critérium de l'action rassasiante d'un aliment et des besoins de la vache pour être rassasiée la notion de ballast telle qu'elle a été définie par LEHMAnN. Celui-ci affirma en effet, que le ballast absorbé par une vache de $500 \mathrm{~kg}$ était une constante d'une valeur de $4,3 \mathrm{~kg}$. Dans le cas où cette conception de LEHMANN se serait révélée fausse, il s'agirait de savoir si un autre élément de mesure permettrait de mieux juger de la question du rassasiement. 
On aboutit aux constatations suivantes:

ro La teneur en ballast ne suffit pas pour déterminer les qualités rassasiantes d'un aliment. Nos essais montrent que la quantité de ballast absorbée n'est pas une constante et que, le chiffre de $4,3 \mathrm{~kg}$ de ballast absorbé n'est pas un chiffre exact.

$2^{\circ}$ La matière sèche non digestible absorbée n'est pas également un critérium sûr pour déterminer le rassasiement.

$3^{0}$ Nos essais, et en outre 1'examen des processus digestifs chez les ruminants montrent que la matière sèche organique absorbée est l'élément le plus sûr pour juger de l'action rassasiante d'un aliment et des besoins de la vache pour être rassasiée.

$4^{0}$ L'élément qui a le plus d'influence sur la quantité de matière sèche absorbée est le bon goût de l'aliment, autrement dit si une vache mange volontiers un aliment qui lui plaît, elle absorbera une quantité de matière sèche relativement plus grande.

$5^{\circ} \mathrm{La}$ quantité de matière sèche absorbée n'est pas influencée par le fait que l'aliment est riche en eau (fourrages verts; racines) ou pauvre en eau (foin sec, paille).

$6^{\circ}$ Les chiffres de matière sèche absorbée constatés au cours de ces essais concordent bien avec ceux indiqués par KELLNER.

$7^{\circ}$ L'importance pour la vache $d u$ bon goût de l'aliment est soulignée par le fait que la quantité de substances digestibles absorbées est fortement augmentée si la vache mange volontiers l'aliment.

Les présents essais et les conclusions qui en ressortent peuvent servir de point de départ à d'autres études sur le même sujet. Si on veut couvrir la plus grande partie des besoins de la vache en substances nutritives avec les aliments produits à la ferme, il est en effet, indispensable de savoir si ces aliments sont mangés volontiers ou non par la vache. La question d'un bon goût apprécié par la vache, joue en effet un rôle important dans l'établissement d'une ration équilibrée et satisfaisante. Il serait intéressant de voir si le goût de l'aliment est influencé par le moment de la récolte (époque de la coupe de la plante utilisée pour le fourrage sec ou l'ensilage) ou par l'apport d'aliments complémentaires (sucre fourrager, mélasse, etc...).

Au cours de nos essais, nous n'avons pas pu éclaircir les questions suivantes :

- relation entre le poids vif de l'animal et la quantité de matière sèche absorbée, influence réciproque de la production de lait et de la quantité de matière sèche absorbée, influence de l'avancement de la gestation sur la quantité de matière sèche absorbée. I1 serait souhaitable que des essais ultérieurs éclairent ces questions.

Je remercie le Professeur Docteur BüNGER, directeur de l'Institut de Kiel, pour son aide et ses conseils. 


\section{BIBLIOGRAPHIE}

(1) Bischoff, Voit. - Gesetze der Ernährung des Fleischfressers, durch neue Untersuchungen festgestellt, i 860 .

(2) Boutrlour (R.). - Dairy Cow Management. (Royal Agr. Coll. issued by the Royal Agric. Coll. Cirencester, 1934).

(3) Boussignault (S.). - Zitiert nach Werner : "Die Bedeutung des Futtervolumens für die Tierernährungslehre ". Forschungsdients, Sonderheft 2, $193^{6}$.

(4) BRUGGeManN (HI.). - - Ein Beitrag zur Futtervolumenfrage bei wachsenden Rindern. Tierernährung Bd. 10,1938 , S. 296.

(5) Bucher (E.). - Einführung in das Studium der Warmluft-Junggrastrocknung im Lichte englischer Erfahrungen. (Landwirtsch. Vorträge. Herausg. Schweiz. Verband d. Lehrer a. landwrirtsch Schulen ud. Ingenieur-Agronomen, Heft $14:$ Die künstliche Grastrocknung, I938).

(6) BüNGer (H.). - Das l'uttervolumen beim Rind. (Forschungsdienst, Sonderheft 8, 1938, S. 506$)$.

(7) - Die Ballastfrage in der Gellügeltütterung. (IDtsch. Landiøirtsch. Gefliggel-Ztg. 4I Jahrg., 1938, S. 531).

(8) - Das Ballastropblem in der Ceflügelhaltung (Kongressbericht des VI. WeltgeflügelKongr., 1936, I. S. 24.3).

(9) - Vereinfachte Futterberechnung nach Milcherzeugungswerten. (Flugbl. Instil. f. Milcherzeug. Pr. Vers. u. Forsch-Anst. f. Milchwirtsch., Kiel, 1939).

(10) DavenporT: - Uber die Beudeuntung Physiologischer Studien ain Tierkörper, Versuch der Wiederkäuerernährung ohne Rauhfutter. (Univ. Illilois Agric. Exp. Stat. Bull., 46, 1897, S. 362, zit. Biedermanss Central-Bl. f. Agricultur-chemie 27, Jahrg., 1898).

(II) DEICKE (J.). - Tierzucht und Fütterung in der Landwirtschaft der Vereinigten Staaaten von Nordamerika. (Ber. ï. LdW.N. F. Sonderheft, igz9).

(i z) Disseliorst (R.) und Mangold (E.). - Anatomie und Physiologie der Haussäugetiere 6 Aufl., i 931.

(i 3) Ellenberger (W.) und Scheunert (A.). - Verfleichende Physiologie der Haussäugetiere 2, Aufl., I 920 .

(14) FALK (H.). - V. Zucht und Fütterung von Milchvieh (Grimmer W. Weigmann, II. Winkler W., Handbuch der Milchwirtschaft Bd. I, I930).

(15) - Die Fütterung und Haltung der Kühe des Deutschen Rinderleistungsbuches, I93 I.

(16) Fingerling (G.). - Die Ernährung der landwirtschaftlichen Haustiere. (Handbuch der Landwirtschaft Bd. 4, I929).

(i 7) Gohres (V.). - Die Naturgesetze der Fütterung der landwirtschaftlichen Haustiere, 1872 .

(I8) Grouven. - Vorträge über Agrikulturchemie mit besonderer Rücksicht auf Tierphysiologie, I860.

(ig) Hagemann (O.). - Anatomie und Physiologie der Huassäugetiere, 2 Bd, Physiologie, I923.

(20) Hansen (J.). - Die Fütterung der Milchkühe. (Ther-bibliothek Bd. I21, 1933).

(21) - Lehrbuch der Rinderzucht, 1927.

(22) Hansson (N.). - Fütterung der Haustiere, 2. Aufl., igz9.

(23) Hansson. - Den forcerade hö-och ensilageutfodringen technik. (Die forcierte Heu und Silagefütterungstechnik) (Landtmannen, 1939).

(24) Henneberg (W.) und Stohmann (F.). - Beiträge zur Begründung einer rationellen Fütterungslehre der Wiederkäuer, Heft I, I860, Heft 2, I863.

(25) Honcamp (F.). - Landwirtschaftliche Fütterungslehre und Futtermittelkunde, I93ı.

(26) Kellner (O.). - Die Ernährung der landwirtschaftlichen Nutztiere. 6, Aufl., igI2.

(27) Kirsch (W.) und Werner (A.). - Flugblätter des Reichnährstandes, Nr 26, 1933.

(28) Krafft (H.). - - Die Tierzuchtlehre, in. Aufl., ig20.

(29) Kronacher (C.). - Allgemeine Tierzucht. 2. Aufl., 1922.

(30) KüHn (J.). - Die Zweckmähigste Ernährung des Rindviehs. 22 Aufl., rgo6.

(3I) - Die Futter und Streunot betreffend. (Mitteil. des landwirtsch. Inst. d. Univers. Halle), Zit. n. Müller, Th. (42). 
(32) Lehmann (F.). - Fütterung der Nutztiere nach dem Stande neuerer Erkenntnis (Tiersüchterische Zeitfragen, 1927 , S. $2 \mathrm{I}$ ).

(33) Lehmann. - Fütterungswesen (Neuere Erfahr. a. d. Geb. d. Tierzucht, I907).

(34) Leroy (A. M.). - Das Volumen der Futterration. (Revue de Zoot. La revue des Eleveurs), Jahrg. 3, 1925, S. 299 : Intern. Agric. Wiss. Rundschau, Rom. N. F. Bd. I, I925, S. 1354 .

(35) Liebig (J.). - Die Tierchemie oder die organische Chenie in ihrer Anwendung auf die Physiologie und Pathologie. I. Aufl., i 843 .

(36) Mangold (E.). - Die Verdaulichkeit der Futtermittel in ihrer Abhängigkeit von verschiedenen Einfüssen. (Forschungsdients, Sonderhefst, 2, I935, S. 35).

(37) Mangold (E.). - - Handbuch der Ernährung und des Stoffwechsels der landwirtschaftlichen Nutztiere, 1932.

(38) Meinhold (W.). - Ist es zweckmässig, den Ballastgehalt der Futtermittel bei Futterberechnungen für Milchkùhe zu beachten? (Dtsch. Landw. Tierzuchtn, 1933, 37 Jahrg. S. 502$)$.

(39) Moore (L. A.) und Winter (O. L.). - I)ie Schnelligskeit des Durchgangs von unverdaulichen Stoffen durch den Verdauungstraktus beim Rind. (J. Dairy Sci., Bd ${ }_{i}$, 1934, S. 297).

(40) Mollgand (H.). - Fùtterungslehre des Milchviehes. I929.

(4I) Muray (A.). - - Die Futteraufnahmefähigkeit des Rindviehs. J. Agric. Sci., 1926. vol. I6, S. 574 ff., réf. von Wendt in : Zïhhlungskunde, Göttingen Bd. 2, 1927, Heft 2, S. 105 .

(42) MüLLER (Th.). - Untersuchungen über die Wirkung geringer (raben von Rauhfutter und die Entbehrlichkeit desselben bei der Fütterung des Milchviehs. (Ber. landw. Inst. Univers. Halle a. S. Ileft 19, 1909, S. 43).

(43) Münzberg (H.) und Nolte (O.). - Die Fütterung des Milchviehs im Deutschen Reiche. (Die Tierernährung Bd. 2, I931, S. 7o).

(44) Ротт (L.). - Handbuch der tierischen Ernährung und der landwirtschaftlichen Futtermitte], Bd. I, 1904.

(45) Richter (K.). - Praktische Viehfütterung. (Tierzucht, 1938).

(46) Schmitt (J.), Patow (C.) und Kliescil (J.). - J. Zuchtung, Emährung und Haltung der Landwirtschaftlichen Ilaustiere (Allg. Teil., 1939).

(47) Settegast (H.). - Tierzucht, I 868.

(48) Settegast. - Die landwirtschaftliche fütterungslehre, i 872.

(49) Stohmann (F.). - Uber die Ernährungsvorgänge des milchproduzierenden Tieres $(J$. f. $L d w .$, Bd. 3,1855, S. 455$)$.

(50) Stutzer (A.). - Berechnung der Futterationen. A. Aufl., igio.

(51) Svoboda (H.). - Das Sättigungsproblem bei der Milchviehfùtterung. (XI. Milchwirtschaftlicher Weltkongress, Berlin, I937, Bd. I, S. I68).

(52) THAER (A.). - Einleitung zur Kenntnis der englichen Landwirtschaft und ihrer neueren pratischen und theoretischen Forschritte in Richtung auf Vervollkommung deutscher Landwirtschaft für denkende Landwirte und Cameralisten, 2. Aufl., I801.

(53) Thaer (A.). - Grundsätze der rationellen Landwirtschaft. Bd 4, Aeft 6, i8 I 2.

(54) Werner (A.). - Das Futter und Fùttern des Milchviehs, 1930.

(55) Werner (A.). - Die Bedeutung des Futtervolumens für die T'ierernährungslehre. (Forschungsdienst, Sonderheft 2, I936).

(56) WiтT (M.). - I)ie Rentabilität des Rindviehstalles, I930.

(57) WITT. - Welche Milchleistungen können mit Wirtshaftseigenem Futter erzielt werden ? (Dtsch. Landev. Tierzucht, 40 Jahrg., I 936, S. 468).

(58) WIтT (M.). - Nährstoffgehalt oder Verdrängungswert der Futtermittel. (Dtsch. Landz'. Tiersucht, 40 Jahrg., 1936, S. 617).

(59) WolfF (E.). - Die Ernährung der landwirtschaftlichen Nutztiere, i 876.

(6o) WolfF (E.). - Rationnelle Fùtterung der landwirtschaftlichen Nutztiere auf Grundlage der neueren physiologischen Feststellungen. 7. Aufl., I899. 